\title{
Long-range corrected exchange-correlation kernels to describe excitons in second-harmonic generation
}

\author{
Nicolas Gauriot* \\ Laboratoire de Chimie Théorique, \\ Sorbonne Université and CNRS, F-75005 Paris, France \\ Valérie Véniard \\ Laboratoire des Solides Irradiés, Ecole Polytechnique, \\ CNRS (UMR 7642), CEA-DSM-IRAMIS, \\ Institut Polytechnique de Paris, 91128 PALAISEAU cedex, \\ France and European Theoretical Spectroscopy Facility (ETSF), France
}

\author{
Eleonora Luppi ${ }^{\dagger}$ \\ Laboratoire de Chimie Théorique, Sorbonne Université and CNRS, F-75005 Paris, France
}

We investigate the role of excitons in second-harmonic generation through the long-range corrected exchange-correlation kernels : empirical LRC, Bootstrap, and jellium-with-a-gap model. We calculate the macroscopic second-order frequency-dependent susceptibility $\chi^{(2)}$. We also present the frequency-dependent macroscopic dielectric function $\epsilon_{\mathrm{M}}$ which is a fundamental quantity in the theoretical derivation of $\chi^{(2)}$. We assess the role of the long-range kernels in describing excitons in materials with different symmetry types : cubic zincblende, hexagonal wurtzite and tetragonal symmetry. Our studies indicate that excitons play an important role in $\chi^{(2)}$ bringing a strong enhancement of the SHG signal. Moreover, we found that the SHG enhancement follows a simple trend determined by the magnitude of the long-range corrected $\alpha$-parameter. This trend is material dependent.

\footnotetext{
* Present address: Cavendish Laboratory, University of Cambridge, J.J. Thompson Avenue, CB3 0HE, Cambridge, United Kingdom

$\dagger$ Corresponding author: eleonora.luppi@lct.jussieu.fr
} 


\section{INTRODUCTION}

Second-harmonic generation (SHG) spectroscopy is a powerful and versatile technique used to probe the properties of matter [1-4]. In particular, in the recent years, SHG has been efficiently used to investigate the role of excitons in different types of materials [4-11]. In fact, excitons lead to sharp resonances in SHG spectra, which are systematically influenced by magnetic and electric fields, strain and temperature [12-17]. Therefore, understanding the role that excitons play in SHG is of great interest for fundamental research and for technological applications.

Excitons are usually described as bound electron-hole pairs, i.e., as an effective two-particle system. They can be classified as Frenkel excitons (bound) which are localised excitations at the atomic sites and Mott-Wannier excitons (continuum), which, instead, are delocalised excitations over the atomic unit cells. These types of excitons have been clearly probed by $\mathrm{SHG}$ in $\mathrm{C}_{60}$ molecular crystals and in $\mathrm{CuCl}$ films [5]. Exciton resonances have also been studied with SHG in bulk GaAs semiconductors [18], bulk hexagonal wide-band-gap semiconductor ZnO [6], as well as in two-dimensional $\mathrm{SiC}, \mathrm{ZnO}$ and GaN hexagonal crystals [19]. Other materials which have been largely investigated in the recent years for their important excitonic properties are two-dimensional (2D) layered materials such as WSe ${ }_{2}$, $\mathrm{WS}_{2}[7-9,20], \mathrm{MoS}_{2}[11]$ and $h$-BN [10].

The quantity that describes the many-electron interactions in the SHG process is the macroscopic second-order susceptibility $\chi^{(2)}[1,21,22] \cdot \chi^{(2)}$ takes into account the variation of the screening fields on the microscopic scale, i.e., crystal local-field effects and electron-hole interactions [22-24]. Chang et al. [25] and Leitsman et al. [26] presented an ab initio many-body formalism for computing the frequency-dependent second-harmonic generation of semiconductor materials including crystal local-field and excitons. In their method the electron-hole interaction is described through the solution of an effective two-particle Hamiltonian, derived from the Bethe-Salpeter equation (BSE) which is very successful in describing excitonic effects in the linear optical response [27-29]. Riefer and Schmidt [30] extended the theory of Leitsmann et al. [26] to the limit $\omega \rightarrow 0$. They used this formalism to calculate SHG spectrum of ZnS, ZnSe, and ZnTe. Attaccalite and Grüning [31] presented another derivation of $\chi^{(2)}$ based on the Berry phase formulation of the dynamical polarisation [32]. They derived the equations of motion describing electron dynamics in an external electric field. Their formalism takes into account the quasi-particle effects, the crystal local fields and the excitons. Luppi et al. $[22,33]$ developed an approach which permits to calculate the macroscopic second-order susceptibility $\chi^{(2)}$ in the framework of Time-Dependent Density-Functional Theory (TDDFT) [34-36]. This approach allows the inclusion of crystal local-fields and excitonic effects through the exchange-correlation kernels $f_{\mathrm{xc}}$ and $g_{x c}[22]$. The kernel $f_{\mathrm{xc}}$ is the same kernel as for the linear response TDDFT, while the kernel $g_{x c}$ is specific to the second-order TDDFT $[22,36]$. An estimation of the importance of $g_{x c}$ has been given in [37] for the static $\chi^{(2)}$. It was shown that the effect of $g_{x c}$ can be of the same order of magnitude as the crystal local-fields. However, the importance of the many-body effects strongly varies with respect to the energy range where $\chi^{(2)}$ is calculated. Despite this investigation, to our knowledge, no other theoretical development exists for $g_{x c}$ which is then neglected in the calculations of the frequency-dependent $\chi^{(2)}$.

In their work, Luppi et al $[22,33]$ investigated the role of excitons in bulk semiconductors demonstrating the importance of long-range interactions to describe excitons in SHG. They used the long range-corrected (LRC) exchange- 
correlation kernel $f_{\mathrm{xc}}=-\alpha^{\mathrm{LRC}} / q^{2}$, which is an empirical kernel that requires a material dependent parameter $\alpha^{\mathrm{LRC}}$ [38]. The LRC kernel was first used in the framework of the linear response theory where the $\alpha^{\mathrm{LRC}}$ was obtained by comparison with the results of the Bethe-Salpeter equation [38]. In this context, the LRC kernel was shown to be able to describe continuum excitons [39]. Note that a different ansatz was also proposed in [37] for the derivation of the long-range kernel, based on the polarization-dependent density-functional theory, giving values close to the one obtained in [38].

Since then, a number of nonempirical exchange-correlation kernels, that have been corrected for the long-range interactions, have been proposed in the literature. The performance of these kernels have been assessed in the linear response, focusing on optical spectra and exciton binding energies [40-43]. Sharma et al. [40] proposed a self-consistent Bootstrap (BO) kernel for the description of excitons in insulators and semiconductors. Later, Rigamonti et al. [41] derived a scalar Bootstrap kernel in RPA (RPA-BOh), which was also applied to bound and continuum excitons for semiconductors. Moreover, they also presented an alternative way to calculate the head of the kernel of Sharma et al. (BOh) [40], which does not require any self-consistency [41]. Trevisanutto et al. [42] proposed an exchange-correlation kernel corrected for the long-range interactions based on the jellium-with-gap model (JGM). However, it was shown that it is not possible to get an accurate exciton binding energy and an optical spectrum in good agreement with experiments at the same time. In the following, we focus only on the optical properties of semiconductors.

In this work, we considered these long-range corrected exchange-correlation kernels and assessed their behaviour, focusing on excitons in SHG spectra. The paper is organized as follows: in Sec. II, we present the general theory for SHG in TDDFT and the long-range kernels which we will use to describe excitons, and in Sec. III we present and discuss the results of the kernels on materials with cubic zincblende, hexagonal wurtzite and tetragonal symmetry. Together with $\chi^{(2)}$, we also investigated the real and the imaginary part of the frequency dependent macroscopic dielectric function $\epsilon_{M}$, which is a fundamental element in the derivation of $\chi^{(2)}$.

\section{THEORY}

\section{A. SHG and symmetries}

The macroscopic second-order susceptibility in TDDFT is $[22,33]$

$$
\begin{array}{r}
\chi_{\alpha \beta \gamma}^{(2)}(\omega, 2 \omega)=\mathrm{N} \epsilon_{\mathrm{M}}^{\mathrm{LL}}\left(\hat{\mathbf{q}}_{3}, 2 \omega\right) \epsilon_{\mathrm{M}}^{\mathrm{LL}}\left(\hat{\mathbf{q}}_{2}, \omega\right) \times \\
\epsilon_{\mathrm{M}}^{\mathrm{LL}}\left(\hat{\mathbf{q}}_{1}, \omega\right) \chi_{\rho \rho \rho}^{(2)}\left(\hat{\mathbf{q}}_{3}, \hat{\mathbf{q}}_{2}, \hat{\mathbf{q}}_{1}, \omega, 2 \omega\right),
\end{array}
$$

where $\alpha, \beta$ and $\gamma$ indicate a specific component of the susceptibility tensor and the normalised wave-vectors $\hat{\mathbf{q}}_{1}$ and $\hat{\mathbf{q}}_{2}$ are defined through a linear combination of the cartesian vectors $\mathrm{x}=(1,0,0), \mathrm{y}=(0,1,0)$ and $\mathrm{z}=(0,0,1)$. This linear combination depends on the symmetries of the system as will be shown later. The normalised wave-vector $\hat{\mathbf{q}}_{3}$ is simply calculated from the sum of $\hat{\mathbf{q}}_{1}$ and $\hat{\mathbf{q}}_{2}$. The longitudinal macroscopic dielectric function is defined as 
$\epsilon_{\mathrm{M}}^{\mathrm{LL}}(\hat{\mathbf{q}}, \omega)=\hat{\mathbf{q}} \cdot \epsilon_{\mathrm{M}}(\omega) \cdot \hat{\mathbf{q}}$ and finally, $\mathrm{N}$ is a constant which also depends on the symmetry of the system studied [22, 33].

\section{Cubic zinc blende symmetry}

For this crystalline system, the only non-zero component is $\chi_{\mathrm{xyz}}^{(2)}$, where $\alpha=\mathrm{x}, \beta=\mathrm{y}$ and $\gamma=\mathrm{z}$. The normalised wave-vectors are defined as $\hat{\mathbf{q}}_{1}=\hat{\mathbf{q}}_{2}=(1 / \sqrt{3}, 1 / \sqrt{3}, 1 / \sqrt{3})$. The symmetry dependent constant is $N=-i \sqrt{3} / 4$ $[22,33]$.

\section{Hexagonal symmetry}

A non-zero component of this crystalline system is, $\chi_{\mathrm{xzx}}^{(2)}$ where $\alpha=\mathrm{x}, \beta=\mathrm{z}$ and $\gamma=\mathrm{x}$. The normalised wavevectors are defined as $\hat{\mathbf{q}}_{1}=(1,0,0)$ and $\hat{\mathbf{q}}_{2}=(0,0,1)$. The symmetry dependent constant is $N=-i / \sqrt{2}$. Another non-zero component is $\chi_{\mathrm{zzz}}^{(2)}$, where $\alpha=\mathrm{z}, \beta=\mathrm{z}$ and $\gamma=\mathrm{z}$. The symmetry dependent constant is $N=-i / 2[44]$.

\section{Tetragonal symmetry}

In this case, a non-zero component is $\chi_{\mathrm{xyz}}^{(2)}$, where $\alpha=\mathrm{x}, \beta=\mathrm{y}$ and $\gamma=\mathrm{z}$. The normalised wave-vectors are defined as $\hat{\mathbf{q}}_{1}=(1 / \sqrt{2}, 1 / \sqrt{2}, 0)$ and $\hat{\mathbf{q}}_{2}=(0,0,1)$. The symmetry dependent constant is $\mathrm{N}=-\mathrm{i} / \sqrt{2}[22]$.

\section{B. Calculation of SHG}

\section{Macroscopic dielectric tensor $\epsilon_{M}(\omega)$}

The macroscopic dielectric tensor $\epsilon_{\mathrm{M}}(\omega)$ is defined as

$$
\epsilon_{\mathrm{M}}(\omega)=\lim _{\mathbf{q} \rightarrow 0} \frac{1}{\epsilon_{\mathbf{G}_{1}=0, \mathbf{G}_{2}=0,}^{-1}(\mathbf{q}, \omega)}
$$

where $\epsilon_{\mathbf{G}_{1}, \mathbf{G}_{2}}^{-1}(\mathbf{q}, \omega)$ is the inverse microscopic dielectric matrix written in terms of the reciprocal-space lattice vectors $\mathbf{G}_{1}$ and $\mathbf{G}_{2}$ for a given wave-vector $\mathbf{q}$ and frequency $\omega$. The case $\mathbf{G}_{1}=\mathbf{G}_{2}=0$ indicates the head element of the inverse microscopic dielectric matrix.

The definition of the inverse microscopic dielectric matrix is

$$
\epsilon_{\mathbf{G}_{1} \mathbf{G}_{2}}^{-1}(\mathbf{q}, \omega)=\delta_{\mathbf{G}_{1} \mathbf{G}_{2}}+\sum_{\mathbf{G}_{3}} v_{\mathbf{G}_{1}} \delta_{\mathbf{G}_{1} \mathbf{G}_{3}} \chi_{\rho \rho, \mathbf{G}_{3} \mathbf{G}_{2}}^{(1)}(\mathbf{q}, \omega)
$$


where $v$ is the Coulomb potential and $\chi_{\rho \rho}^{(1)}$ is the linear response matrix which can be obtained by solving the TDDFT equation :

$$
\begin{array}{r}
\chi_{\rho \rho, \mathbf{G}_{1} \mathbf{G}_{2}}^{(1)}(\mathbf{q}, \omega)=\chi_{0, \mathbf{G}_{1} \mathbf{G}_{2}}^{(1)}(\mathbf{q}, \omega)+ \\
\sum_{\mathbf{G}_{3} \mathbf{G}_{4}} \chi_{0, \mathbf{G}_{1} \mathbf{G}_{3}}^{(1)}(\mathbf{q}, \omega) f_{\mathrm{Hxc}, \mathbf{G}_{3} \mathbf{G}_{4}}(\mathbf{q}, \omega) \chi_{\rho \rho, \mathbf{G}_{4} \mathbf{G}_{2}}^{(1)}(\mathbf{q}, \omega),
\end{array}
$$

where $\chi_{0}^{(1)}$ is the independent-particle response matrix and $f_{\mathrm{Hxc}}=v+f_{\mathrm{xc}}$ is the sum of the Coulomb potential $v$ and of the exchange-correlation kernel $f_{\mathrm{xc}}$.

\section{Second-order response function $\chi_{\rho \rho \rho}^{(2)}$}

The second-order response function $\chi_{\rho \rho \rho}^{(2)}$ in TDDFT is

$$
\begin{aligned}
& \sum_{\mathbf{G}_{5}}\left[\delta_{\mathbf{G}_{1}, \mathbf{G}_{5}}-\sum_{\mathbf{G}_{4}} \chi_{0, \mathbf{G}_{1} \mathbf{G}_{4}}^{(1)}\left(\mathbf{q}_{3}, 2 \omega\right) f_{\mathrm{Hxc}, \mathbf{G}_{4} \mathbf{G}_{5}}\left(\mathbf{q}_{3}, 2 \omega\right)\right] \chi_{\rho \rho \rho}^{(2)}, \mathbf{G}_{5} \mathbf{G}_{2} \mathbf{G}_{3}\left(\mathbf{q}_{3}, \mathbf{q}_{2}, \mathbf{q}_{1}, \omega\right)=\sum_{\mathbf{G}_{4} \mathbf{G}_{6}} \chi_{0, \mathbf{G}_{1} \mathbf{G}_{4} \mathbf{G}_{6}}^{(2)}\left(\mathbf{q}_{3}, \mathbf{q}_{2}, \mathbf{q}_{1}, 2 \omega\right) \times \\
& {\left[\delta_{\mathbf{G}_{6} \mathbf{G}_{3}}+\sum_{\mathbf{G}_{7}} f_{\mathrm{Hxc}, \mathbf{G}_{6} \mathbf{G}_{7}}\left(\mathbf{q}_{2}, \omega\right) \chi_{\rho \rho, \mathbf{G}_{7} \mathbf{G}_{3}}^{(1)}\left(\mathbf{q}_{2}, \omega\right)\right]\left[\delta_{\mathbf{G}_{2} \mathbf{G}_{4}}+\sum_{\mathbf{G}_{8}} f_{\mathrm{Hxc}, \mathbf{G}_{2} \mathbf{G}_{8}}\left(\mathbf{q}_{1}, \omega\right) \chi_{\rho \rho, \mathbf{G}_{8} \mathbf{G}_{4}}^{(1)}\left(\mathbf{q}_{1}, \omega\right)\right]+}
\end{aligned}
$$

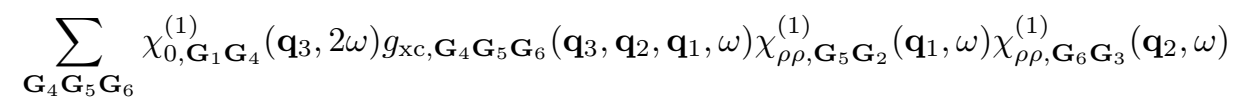

which depends on $f_{\mathrm{Hxc}}$, on the second-order kernel $g_{\mathrm{xc}}$, on the linear response matrices $\chi_{0}^{(1)}, \chi_{\rho \rho}^{(1)}$ and on the independent-particle second-order response matrix $\chi_{0}^{(2)}[22,33]$.

\section{Exchange-correlation kernels}

In our study we considered the LRC kernel [38, 39], the BO kernel, i.e., the BOh [40, 41], the RPA-BOh kernel [41] and the JGM kernel [42] . Despite the different derivations of these kernels, their role is to screen the Coulomb potential by simulating the Coulomb repulsion between the electron-hole pair. No exchange contribution is included in these kernels. Moreover, in all of our calculations, we included a scissor shift to simulate the quasiparticle effects and we considered only the head contribution of the long-range corrected kernels.

\section{1. $L R C$}

The empirical LRC kernel is defined as [38, 39]

$$
f_{\mathrm{xc}, \mathbf{G G}}^{\mathrm{LRC}}(\mathbf{q})=-\frac{\alpha^{\mathrm{LRC}}}{\left|\mathbf{q}+\mathbf{G}^{\prime}\right|^{2}} \delta\left(\mathbf{G}^{\prime}-\mathbf{G}\right)
$$


and the head-only term of the LRC kernel is $f_{\mathrm{xc}}^{\mathrm{LRC}}(\mathbf{q})=-\alpha^{\mathrm{LRC}} /|\mathbf{q}|^{2}$. The parameter $\alpha^{\mathrm{LRC}}=C_{1} / \epsilon_{\infty}-C_{2}$ is expressed in terms of the constants $C_{1}=4.615, C_{2}=0.213$ and of the experimental high-frequency dielectric constant $\epsilon_{\infty}$. The values of $C_{1}$ and $C_{2}$ have been obtained through a fit, which uses the theoretical lattice constants of the materials. This parametrisation can also be used with the experimental lattice constants [22, 43].

This kernel has demonstrated to be able to simulate continuum excitons in the absorption spectrum of materials when quasiparticle energies or a scissor shift are used [38, 39]. Luppi et al. [22, 33] also used this kernel for the calculation of SHG in bulk semiconductors. In particular, they obtained a remarkable agreement in the experimental intensity of the highest peak of the second-order susceptibility for GaAs [33]. This result showed the importance of long-range interactions in SHG spectra. However, there are still some discrepancies between the experimental and the theoretical SHG spectra. These can be due to the crude approximation of the parameter $\alpha^{\mathrm{LRC}}$ which is a mean value of the dynamical dependence of $f_{\mathrm{xc}}$ in a given range of frequency.

\section{2. $J G M$}

Constantin et al. [45] proposed a dynamic exchange-correlation kernel of the uniform electron gas. In order to describe the short-range interactions, they defined their kernel by introducing an effective interaction in the form of a frequency-dependent error-function, which reduces the effects of the electron Coulomb interaction. Then, they imposed the fulfillments of the compressibility and the third-frequency-moment sum rule as well as the correct asymptotic behaviour at large wave vectors. Finally, they found an accurate exchange-correlation kernel that reproduces the wave-vector analysis and the imaginary frequency analysis of the correlation energy of the uniform electron gas. Trevisanutto et al. [42] extended this kernel, in the static case, to the jellium with gap model (JGM) which permits to treat weak excitonic effects in semiconductors and bound excitons in insulators.

This matrix form of the JGM kernel in reciprocal space can be decomposed as a sum of three independent terms as follows :

$$
f_{\mathrm{xc}, \mathbf{G G}}^{\mathrm{JGM}}(\mathbf{q})=f_{\mathrm{xc}, \mathbf{G} \mathbf{G}^{\prime}}^{\mathrm{JGM} 1}(\mathbf{q})+f_{\mathrm{xc}, \mathbf{G} \mathbf{G}^{\prime}}^{\mathrm{JGM} 2}(\mathbf{q})+f_{\mathrm{xc}, \mathbf{G} \mathbf{G}^{\prime}}^{\mathrm{JGM} 3}(\mathbf{q}) .
$$

The first term is

$$
f_{\mathrm{xc}, \mathbf{G G}}^{\mathrm{JGM} 1}(\mathbf{q})=-\frac{4 \pi B^{\prime}\left(\mathbf{G}-\mathbf{G}^{\prime}\right)}{\left|\mathbf{q}+\mathbf{G}^{\prime}\right|^{2}}
$$

where

$$
B^{\prime}\left(\mathbf{G}-\mathbf{G}^{\prime}\right)=\int \mathrm{d} \mathbf{r} e^{-i\left(\mathbf{G}-\mathbf{G}^{\prime}\right) \mathbf{r}} B^{\prime}\left(n(\mathbf{r}), E_{g}\right)
$$


and

$$
B^{\prime}\left(n(\mathbf{r}), E_{g}\right)=\frac{B(n(\mathbf{r}))+E_{g}}{1+E_{g}}
$$

The quantity $E_{g}$ is the minimum electronic energy gap of the material while the quantity $B(n)$ is parametrised as

$$
B(n)=\frac{1+a_{1} r_{s}^{1 / 2}+a_{2} r_{s}^{3 / 2}}{3+b_{1} r_{s}^{1 / 2}+b_{2} r_{s}^{3 / 2}},
$$

where $r_{s}=[3 /(4 \pi n)]^{1 / 3}, a_{1}=2.15, a_{2}=0.435, b_{1}=1.57$ and $b_{2}=0.409$. [45]

The second term is

$$
f_{\mathrm{xc}, \mathbf{G G}^{\prime}}^{\mathrm{JGM} 2}(\mathbf{q})=\frac{4 \pi H\left(\mathbf{G}-\mathbf{G}^{\prime}, \mathbf{G}^{\prime}\right)}{\left|\mathbf{q}+\mathbf{G}^{\prime}\right|^{2}}
$$

where

$$
H\left(\mathbf{G}-\mathbf{G}^{\prime}, \mathbf{G}^{\prime}\right)=\int \mathrm{d} \mathbf{r} e^{-i\left(\mathbf{G}-\mathbf{G}^{\prime}\right) \mathbf{r}} B^{\prime}\left(n(\mathbf{r}), E_{g}\right) e^{-k_{n, E g}^{\prime}\left|\mathbf{q}+\mathbf{G}^{\prime}\right|^{2}}
$$

and

$$
k_{n, E_{g}}^{\prime}=k_{n, E_{g}}+\frac{1}{4 \pi|\mathbf{q}+\mathbf{G}|^{2}} \frac{E_{g}^{2}}{n B^{\prime}\left(n, E_{g}\right)}
$$

with

$$
k_{n, E_{g}}=-\frac{\alpha n^{\beta}}{B^{\prime}\left(n, E_{g}\right)}
$$

where $\alpha=-0.025$ and $\beta=-0.691$. [45]

Finally, the third term is

$$
f_{\mathrm{xc}, \mathbf{G} \mathbf{G}^{\prime}}^{\mathrm{JGM} 3}(\mathbf{q})=-D^{\prime}\left(\mathbf{G}-\mathbf{G}^{\prime}\right) \frac{1}{1+1 /\left|\mathbf{q}+\mathbf{G}^{\prime}\right|^{2}}
$$

where 


$$
D^{\prime}\left(\mathbf{G}-\mathbf{G}^{\prime}\right)=\int \mathrm{d} \mathbf{r} e^{-i\left(\mathbf{G}-\mathbf{G}^{\prime}\right) \mathbf{r}} \frac{4 \pi C^{\prime}\left(n(\mathbf{r}), E_{g}\right)}{k_{F}^{2}}
$$

and

$$
C^{\prime}\left(n(\mathbf{r}), E_{g}\right)=\frac{C(n(\mathbf{r}))}{1+E_{g}} .
$$

The quantity $C(n)$ is parametrised as [45]

$$
C(n)=-\frac{\pi}{2 k_{F}} \frac{d\left(r_{s} \epsilon_{c}\right)}{d r_{s}}
$$

where $k_{F}=\left(3 \pi^{2} n\right)^{1 / 3}$ represents the Fermi momentum of the uniform electron gas and $\epsilon_{c}$ is the correlation energy in the Perdew-Wang parametrization.

Considering only the head of this kernel, it is possible to define a LRC $\alpha$-parameter for the JGM kernel as

$$
\alpha^{\mathrm{JGM}}=\alpha^{\mathrm{JGM} 1}+\alpha^{\mathrm{JGM} 2}+\alpha^{\mathrm{JGM} 3}
$$

where $\alpha^{\mathrm{JGM} 1}=4 \pi B^{\prime}(0), \alpha^{\mathrm{JGM} 2}=4 \pi H(0,0)$ and $\alpha^{\mathrm{JGM} 3}=0$. Clearly $f_{\mathrm{xc}}^{\mathrm{JGM} 3}$ gives no contribution.

\section{Bootstrap}

Sharma et al.[40] proposed a bootstrap kernel for optical spectra of semiconductors and insulators, which reads as

$$
f_{\mathrm{xc}, \mathbf{G G}^{\prime}}^{\mathrm{BO}}(\mathbf{q})=\frac{v_{\mathbf{G}}^{1 / 2}(\mathbf{q}) \epsilon_{\mathbf{G G}^{\prime}}^{-1}(\mathbf{q}, 0) v_{\mathbf{G}^{\prime}}^{1 / 2}}{1-\epsilon_{\mathrm{RPA}, 00}(\mathbf{q}, 0)}
$$

and which is iterated numerically to self-consistency. In this case, it is also possible to define a LRC $\alpha$-parameter

$$
\alpha^{\mathrm{BO}}(\mathbf{q})=-\frac{4 \pi \epsilon_{00}^{-1}(\mathbf{q}, 0)}{1-\epsilon_{\mathrm{RPA}, 00}(\mathbf{q}, 0)} .
$$

Unlike the JGM kernel, the bootstrap kernel depends explicitly on the structure of the material through $\epsilon^{-1}$ and $\epsilon_{\mathrm{RPA}}$, which means that for non-isotropic systems the kernel is different in different directions.

To avoid self-consistency, Rigamonti et al.[41] proposed an alternative but equivalent analytical form for the head of the Sharma et al.[40] boostrap kernel (Eq. (21)), which is 


$$
f_{\mathrm{xc}}^{\mathrm{BOh}}(\mathbf{q})=\frac{1}{\epsilon_{M}(0) \chi_{00}^{0}(\mathbf{q}, 0)}
$$

with

$\epsilon_{M}(0)=\lim _{\mathbf{q} \rightarrow 0} \frac{1}{2}\left(1+\frac{\bar{\chi}_{00}^{\mathrm{RPA}}(\mathbf{q}, 0)}{\chi_{00}^{0}(\mathbf{q}, 0)}-v_{0}(\mathbf{q}) \bar{\chi}_{00}^{\mathrm{RPA}}(\mathbf{q}, 0)\right)+\sqrt{\frac{1}{4}\left(1+\frac{\bar{\chi}_{00}^{\mathrm{RPA}}(\mathbf{q}, 0)}{\chi_{00}^{0}(\mathbf{q}, 0)}-v_{0}(\mathbf{q}) \bar{\chi}_{00}^{\mathrm{RPA}}(\mathbf{q}, 0)\right)^{2}-\frac{\bar{\chi}_{00}^{\mathrm{RPA}}(\mathbf{q}, 0)}{\chi_{00}^{0}(\mathbf{q}, 0)}}$.

Here, $\bar{\chi}_{00}^{\mathrm{RPA}}$ is defined as in Eq. (4), but without the long-range part of the Coulomb interaction $(\bar{v}(\mathbf{G}=\mathbf{0})=\mathbf{0})$,

$$
\begin{array}{r}
\bar{\chi}_{\rho \rho, \mathbf{G}_{1} \mathbf{G}_{2}}^{(1)}(\mathbf{q}, \omega)=\chi_{0, \mathbf{G}_{1} \mathbf{G}_{2}}^{(1)}(\mathbf{q}, \omega)+ \\
\sum_{\mathbf{G}_{3}} \chi_{0, \mathbf{G}_{1} \mathbf{G}_{3}}^{(1)}(\mathbf{q}, \omega) \bar{v}\left(\mathbf{G}_{3}\right) \bar{\chi}_{\rho \rho, \mathbf{G}_{3} \mathbf{G}_{2}}^{(1)}(\mathbf{q}, \omega),
\end{array}
$$

This form brings results which are indistinguishable with the converged iterative results of the BO kernel.

\section{RPA bootstrap}

Rigamonti et al. [41] proposed another kernel defined as

$$
f^{\mathrm{RPA}-\mathrm{BOh}}(\mathbf{q})=\frac{\epsilon_{\mathrm{RPA}, 00}^{-1} v(\mathbf{q})}{1-1 / \epsilon_{\mathrm{RPA}, 00}^{-1}(\mathbf{q}, 0)},
$$

where the LRC $\alpha$-parameter is

$$
\alpha^{\mathrm{RPA}-\mathrm{BOh}}=-\frac{4 \pi \epsilon_{\mathrm{RPA}, 00}^{-1}(0,0)}{1-1 / \epsilon_{\mathrm{RPA}, 00}^{-1}(0,0)} .
$$

This kernel is close to the BO kernel in Eq. (21) but there is no self-consistency condition. Moreover, the derivation of this kernel relies on the assumptions that the kernel is static in the optical range, that the static dielectric constant can be correctly described in RPA and that it is much larger than 1. BO and RPA-BOh give similar results in absorption spectra of semiconductors for weak excitons while noticeable changes are present for strong excitons. Note that an expression similar to Eq. (27) has been obtained in the context of Current-Density-Functional Theory, assuming a small dielectric constant $\epsilon_{\mathrm{RPA}, 00}<2[46]$.

\section{RESULTS}

We calculated $\chi_{\alpha \beta \gamma}^{(2)}(\omega, 2 \omega)$ for i) $\mathrm{SiC}$ and GaAs bulk semiconductors (cubic zinc-blende), ii) polytypes $2 \mathrm{H}-\mathrm{SiC}, 4 \mathrm{H}$ $\mathrm{SiC}, 6 \mathrm{H}-\mathrm{SiC}$ and $\mathrm{ZnO}$ semiconductors (hexagonal wurtzite) and iii) $\mathrm{Zn}_{2} \mathrm{Ge}_{2} \mathrm{P}_{4}$ (tetragonal). We first calculated the 
electronic structure of the materials in their ground-state within Density-Functional Theory (DFT) in the local-density approximation (LDA), using norm-conserving Trouiller-Martins pseudopotentials [47, 48] and plane-wave basis set with the ABINIT code [49-51]. In the case of Gallium, we explicitly included the $3 \mathrm{~d}$ semicore states as valence states, giving the valence configuration $3 d^{10} 4 s^{2} 4 p^{1}[52,53]$. Then, we calculated the optical spectra in TDDFT as implemented in the 2light code [54]. In particular, the TDDFT calculations were always done on top of scissor-corrected LDA band structure, so that only the excitonic part of the exact exchange-correlation kernel $f_{\mathrm{xc}}$ is approximated. We have considered the head-only version of the kernels presented above. The comparison between the head-only and full form of the kernel is shown for $\mathrm{SiC}$ in the Supplemental Information. Moreover, we emphasise that for all the calculations we used $g_{\mathrm{xc}}$ equal to zero. In fact, even if we know that this kernel behaves as $g_{x c}=-\beta / \mathbf{q}^{3}$ where $\beta$ is constant, to our knowledge the value of $\beta$ is still not known.

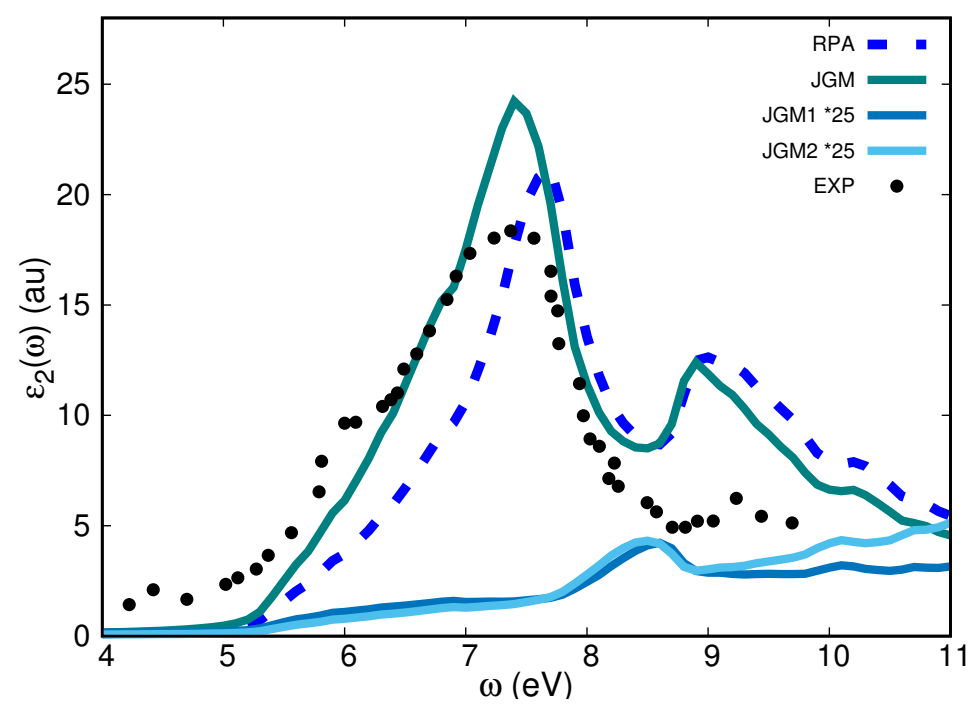

FIG. 1. SiC : $\epsilon_{2}$ calculated in RPA, JGM, JGM1, JGM2 and experiment [55]. JGM1 and JGM2 have been multiplied by 25.

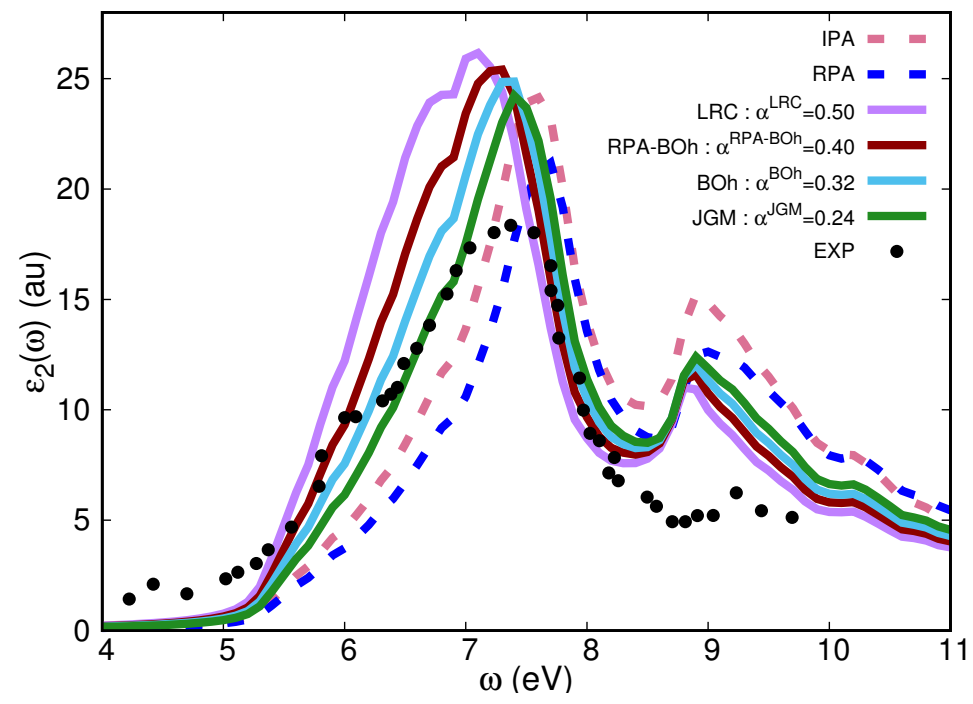

FIG. 2. SiC : $\epsilon_{2}(\omega)$ calculated in IPA, RPA, LRC, RPA-BOh, BOh, JGM kernels and experiment [55]. 


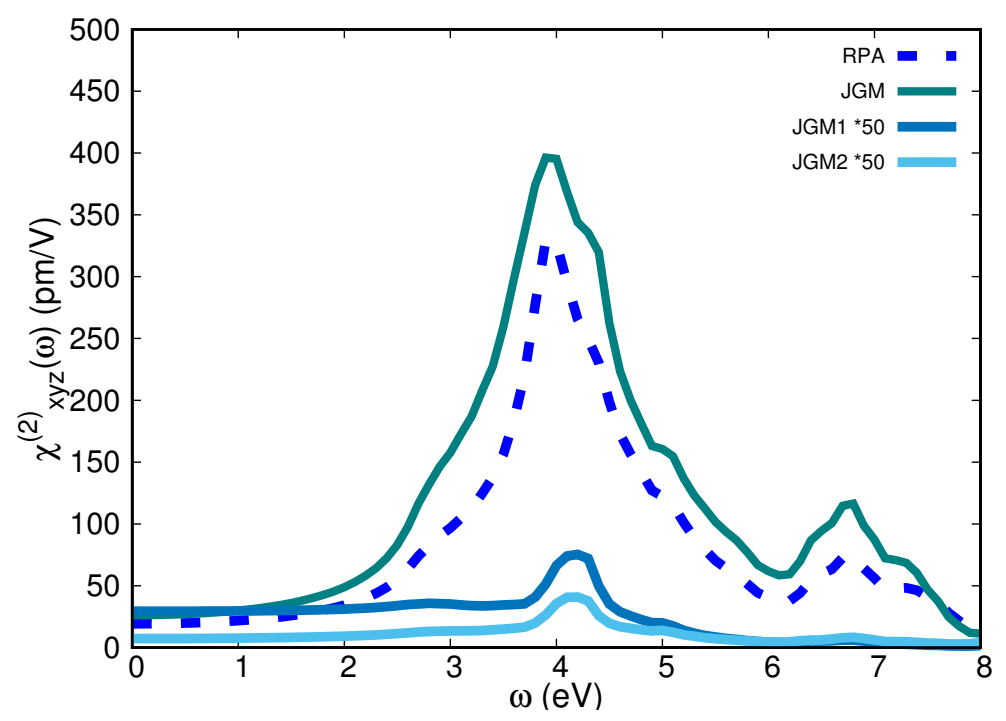

FIG. 3. SiC : $\chi_{\text {xyz }}^{(2)}$ calculated in RPA, JGM, JGM1, JGM2. JGM1 and JGM2 have been multiplied by 50.

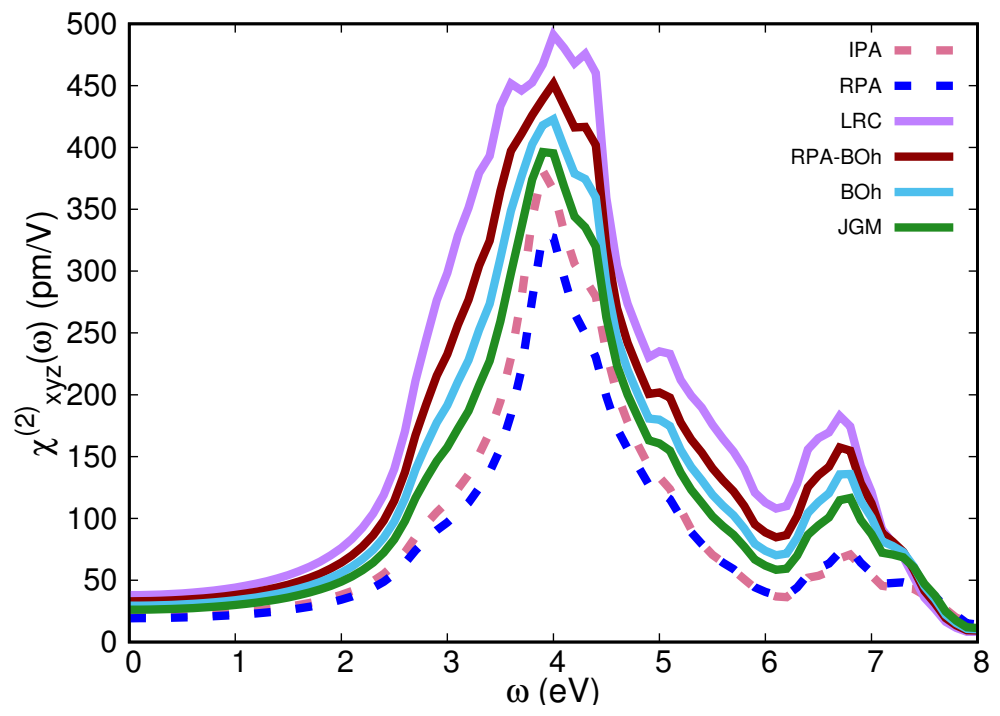

FIG. 4. SiC : $\chi_{\text {xyz }}^{(2)}$ calculated with IPA, RPA, LRC, RPA-BOh, BOh and JGM kernels.

To fully analyse the role of long-range corrected kernels in $\chi_{\alpha \beta \gamma}^{(2)}(\omega, 2 \omega)$ (see Eq. (1)), we also analyse the impact of the long-range interactions on $\epsilon_{\mathrm{M}}$. In fact, in Eq. (1) $\epsilon_{\mathrm{M}}$ enters explicitly in the calculation of $\chi_{\alpha \beta \gamma}^{(2)}(\omega, 2 \omega)$ at frequency $\omega$ and $2 \omega$. As $\epsilon_{\mathrm{M}}=\epsilon_{1}+i \epsilon_{2}$, we analysed both its real and imaginary parts. Concerning the real part $\epsilon_{1}$, we reported here only its static value while the full frequency-dependent spectrum has been reported in the Supplemental Information.

\section{A. Cubic SiC}

We used the experimental lattice constant $4.360 \AA$ and an energy cutoff of 50 Ha [59]. We used 16000 shifted $k$ points in the Brillouin zone (BZ), 16 unoccupied states and $459 \mathbf{G}$ vectors for the wavefunctions. Crystal local-field 


\begin{tabular}{ccccccccc}
\hline \hline & & \multicolumn{1}{c}{$\mathrm{SiC}$} \\
& IPA & RPA & LRC & RPA-BOh & BOh & & \\
\hline$\epsilon_{1}$ & 6.63 & 6.09 & 7.39 & 7.09 & 6.86 & 6.64 & 6.5 \\
$\alpha$ & & & 0.50 & 0.40 & 0.32 & 0.24 & \\
$\left|\chi_{x y z}^{(2)}\right|$ & 21.05 & 19.24 & 37.96 & 32.95 & 29.40 & 26.15 & \\
\hline \hline
\end{tabular}

TABLE I. Static values of $\epsilon_{1}$ and $\left|\chi_{\mathrm{xyz}}^{(2)}\right|[\mathrm{pm} / \mathrm{V}]$ for SiC. The $\alpha$-parameter is reported for the long-range corrected kernels. (a) from Ref. [55]

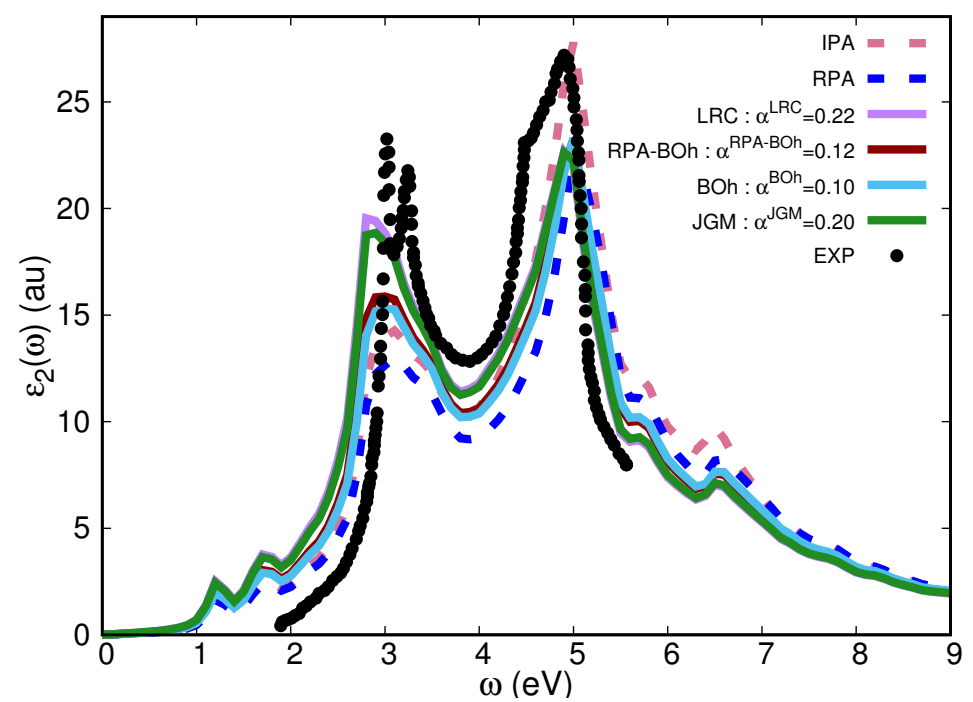

FIG. 5. GaAs : $\epsilon_{2}(\omega)$ calculated with IPA, RPA, LRC, RPA-BOh, BOh, JGM kernels and experiment [56].

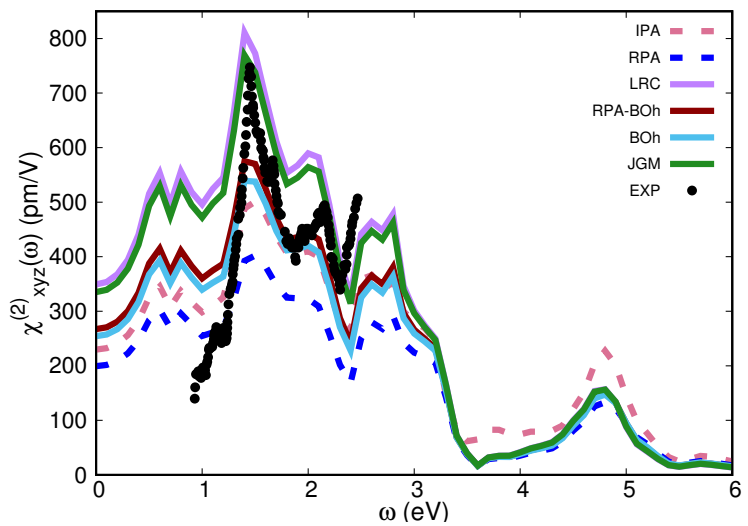

FIG. 6. GaAs : $\chi_{\mathrm{xyz}}^{(2)}$ calculated with IPA, RPA, LRC, RPA-BOh, BOh, JGM kernels and experiment [18].

effects are fully taken into account using 89 G vectors. In order to simulate the quasiparticle energies we used, on top of an LDA band structure, a scissors shift of $0.84 \mathrm{eV}$ [60]. We obtained a minimum electronic gap of $2.17 \mathrm{eV}$ and a direct electronic gap of $5.36 \mathrm{eV}$. In the case of the JGM kernel we used the experimental minimum electronic gap $E_{g}=2.40 \mathrm{eV}$.

In Fig. (1), we show $\epsilon_{2}$ for the JGM kernel for which we used the experimental gap $E_{g}=2.40 \mathrm{eV}$ [61]. Comparing the effect of this kernel with the RPA calculation $\left(f_{\mathrm{xc}}=0\right)$ we observed that the energy position of the main peak is shifted to lower energy and its intensity is increased. Instead, the second peak is almost unaffected by the JGM 


\begin{tabular}{cccccccc}
\hline \hline & & \multicolumn{7}{c}{ GaAs } & & & \\
& IPA & RPA & LRC & RPA-BOh & BOh & JGM & $\operatorname{Exp}^{(a)}$ \\
\hline$\epsilon_{1}$ & 11.80 & 10.73 & 12.73 & 11.73 & 11.55 & 12.57 & 10.6 \\
$\alpha$ & & & 0.22 & 0.12 & 0.10 & 0.20 & \\
$\left|\chi_{x y z}^{(2)}\right|$ & 229.73 & 199.39 & 349.23 & 267.38 & 254.38 & 335.14 & 200 \\
\hline \hline
\end{tabular}

TABLE II. Static values of $\epsilon_{1}$ and $\left|\chi_{\mathrm{xyz}}^{(2)}\right|[\mathrm{pm} / \mathrm{V}]$ for GaAs. The $\alpha$-parameter is reported for the long-range corrected kernels. (a) from Refs. [57] and [58]

\begin{tabular}{|c|c|c|c|c|c|c|}
\hline \multicolumn{7}{|c|}{$2 \mathrm{H}-\mathrm{SiC}$} \\
\hline & IPA & RPA & LRC & RPA-BOh & $\mathrm{BOh}$ & JGM \\
\hline$\epsilon_{1, \mathrm{xx}}$ & 6.65 & 6.13 & 7.58 & 7.13 & 6.91 & 7.32 \\
\hline$\alpha_{\mathrm{xx}}$ & & & 0.54 & 0.40 & 0.32 & 0.46 \\
\hline$\epsilon_{1, \mathrm{zz}}$ & 6.93 & 6.41 & 7.89 & 7.41 & 7.20 & 7.75 \\
\hline$\alpha_{\mathrm{zz}}$ & & & 0.50 & 0.36 & 0.29 & 0.46 \\
\hline$\left|\chi_{\mathrm{xzx}}^{(2)}\right|$ & 12.01 & 11.63 & 24.32 & 19.59 & 17.61 & 22.15 \\
\hline$\left|\chi_{\mathrm{zzz}}^{(2)}\right|$ & 8.80 & 6.57 & 13.59 & 10.92 & 9.86 & 12.75 \\
\hline \multicolumn{7}{|c|}{$4 \mathrm{hSiC}$} \\
\hline & IPA & RPA & LRC & RPA-BOh & $\mathrm{BOh}$ & JGM \\
\hline$\epsilon_{1, \mathrm{xx}}$ & 6.58 & 6.38 & 7.88 & 7.38 & 7.18 & 7.59 \\
\hline$\alpha_{\mathrm{xx}}$ & & & 0.51 & 0.37 & 0.30 & 0.43 \\
\hline$\epsilon_{1, \mathrm{zz}}$ & 6.82 & 6.66 & 8.26 & 7.66 & 7.48 & 8.02 \\
\hline$\alpha_{\mathrm{zz}}$ & & & 0.48 & 0.33 & 0.28 & 0.43 \\
\hline$\left|\chi_{\mathrm{xzX}}^{(2)}\right|$ & 12.17 & 12.07 & 25.18 & 19.89 & 18.21 & 22.59 \\
\hline$\left|\chi_{\mathrm{zzZ}}^{(2)}\right|$ & 17.54 & 15.90 & 33.03 & 25.90 & 23.84 & 30.29 \\
\hline \multicolumn{7}{|c|}{$6 \mathrm{hSiC}$} \\
\hline & IPA & $\mathrm{RPA}$ & LRC & RPA-BOh & $\mathrm{BOh}$ & JGM \\
\hline$\epsilon_{1, \mathrm{xx}}$ & 6.73 & 6.14 & 8.15 & 7.14 & 6.96 & 7.24 \\
\hline$\alpha_{\mathrm{xx}}$ & & & 0.54 & 0.40 & 0.34 & 0.43 \\
\hline$\epsilon_{1, \mathrm{zz}}$ & 6.91 & 6.42 & 8.33 & 7.42 & 7.25 & 7.67 \\
\hline$\alpha_{\mathrm{zz}}$ & & & 0.50 & 0.36 & 0.31 & 0.43 \\
\hline$\left|\chi_{\mathrm{xzx}}^{(2)}\right|$ & 10.02 & 10.02 & 21.08 & 16.95 & 15.58 & 18.41 \\
\hline$\left|\chi_{\mathrm{zzz}}^{(2)}\right|$ & 15.83 & 15.34 & 31.81 & 25.50 & 23.52 & 28.57 \\
\hline
\end{tabular}

TABLE III. Static values of $\epsilon_{1, \mathrm{xx}}, \epsilon_{1, \mathrm{zz}},\left|\chi_{\mathrm{xzx}}^{(2)}\right|[\mathrm{pm} / \mathrm{V}]$ and $\left|\chi_{\mathrm{zzz}}^{(2)}\right|[\mathrm{pm} / \mathrm{V}]$ for $2 \mathrm{H}-\mathrm{SiC}, 4 \mathrm{H}-\mathrm{SiC}$ and $6 \mathrm{H}-\mathrm{SiC}$. The $\alpha$-parameter is reported for the long-range corrected kernels.

kernel. The results are consistent with the theoretical calculation presented by Trevisanutto et al. [42]. However, to deeper understand the JGM behaviour we separately calculate the contribution to $\epsilon_{\mathrm{M}}$ from JGM1 and JGM2. We recall that JGM3 gives no contribution. We observed that in the case of JGM1 and JGM2 the spectrum of $\epsilon_{2}$ is very peculiar as its shape is completely different from the JGM calculation and from experiments. In fact, even if both JGM1 and JGM2 contain the $1 / q^{2}$ dependence, we obtained $\alpha^{\mathrm{JGM} 1}=10.36$ and $\alpha_{\mathrm{xc}}^{J G M 2}=-10.12$, which, in absolute value, is orders of magnitude different than $\alpha^{\mathrm{JGM}}=0.24$.

In Fig. (2) we compared all the long-range corrected kernels studied here with IPA and RPA. The kernels have the same trend which is to shift the energy position of the main peak to lower energy and to increase its intensity keeping the second peak almost unchanged. Moreover, it is possible to quantify the strength of this effect by comparing the $\alpha$ values for each kernels. We have that $\alpha^{\mathrm{LRC}}=0.50>\alpha^{\mathrm{RPA}-\mathrm{BOh}}=0.40>\alpha^{\mathrm{BOh}}=0.32>\alpha^{\mathrm{JGM}}=0.24$. The same trend is found for frequency-dependent $\epsilon_{1}$ (see Supplemental Information) which static value is reported in Tab. (I). 

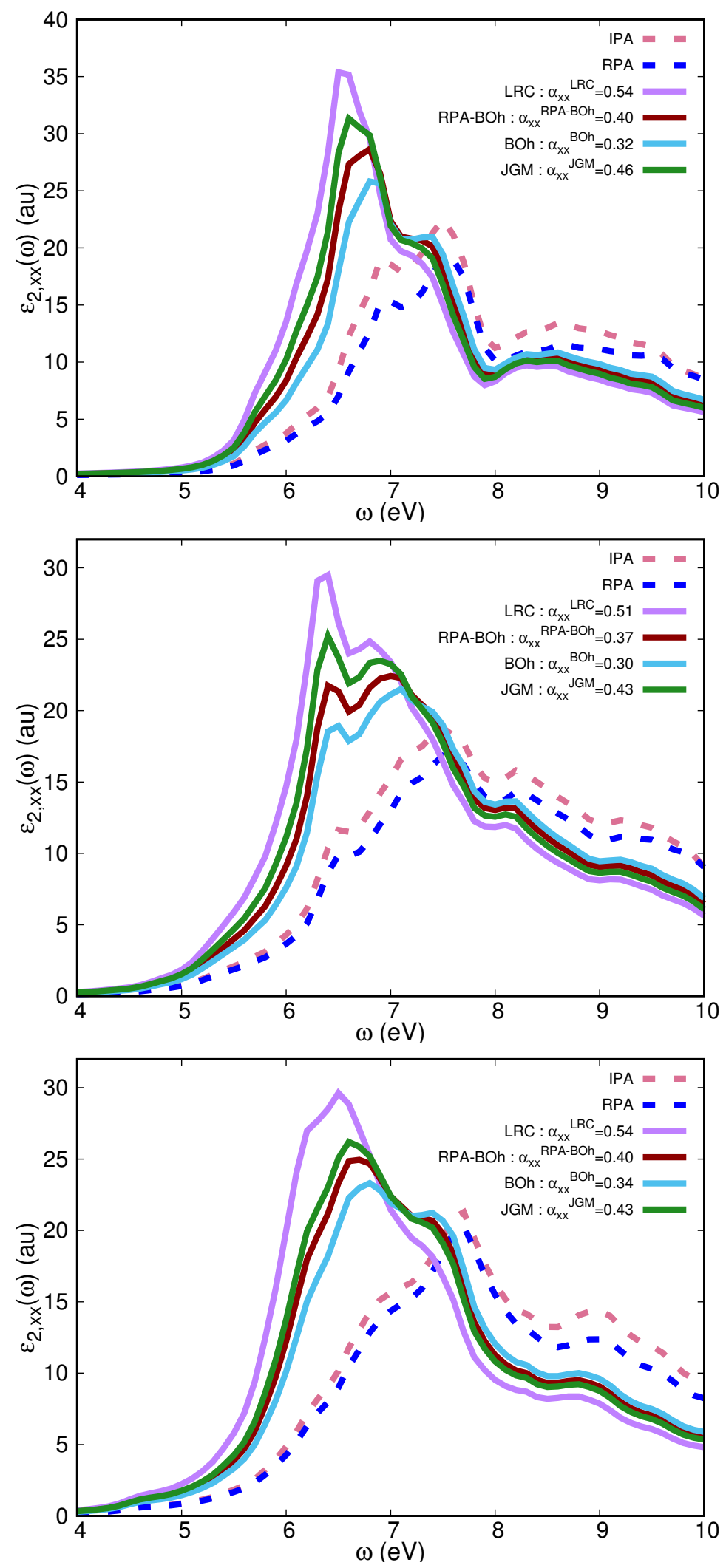

FIG. 7. SiC-2H (top), SiC-4H (center) and SiC-6H (bottom) : $\epsilon_{2, \mathrm{xx}}(\omega)$ calculated with IPA, RPA, LRC, RPA-BOh, BOh, JGM kernels. 

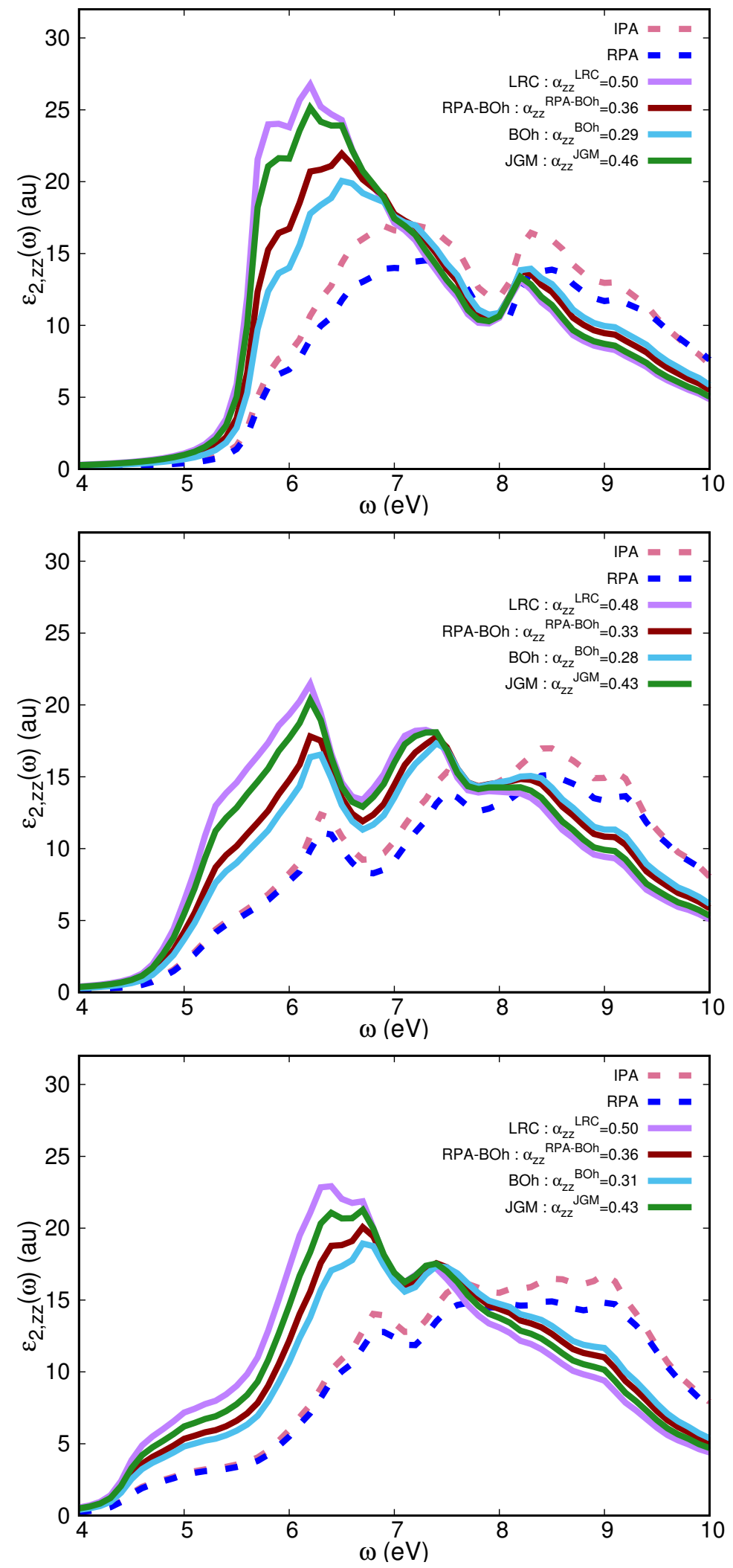

FIG. 8. SiC-2H (top), SiC-4H (center) and SiC-6H (bottom) : $\epsilon_{2, \mathrm{zz}}(\omega)$ calculated with IPA, RPA, LRC, RPA-BOh, BOh, JGM kernels. 

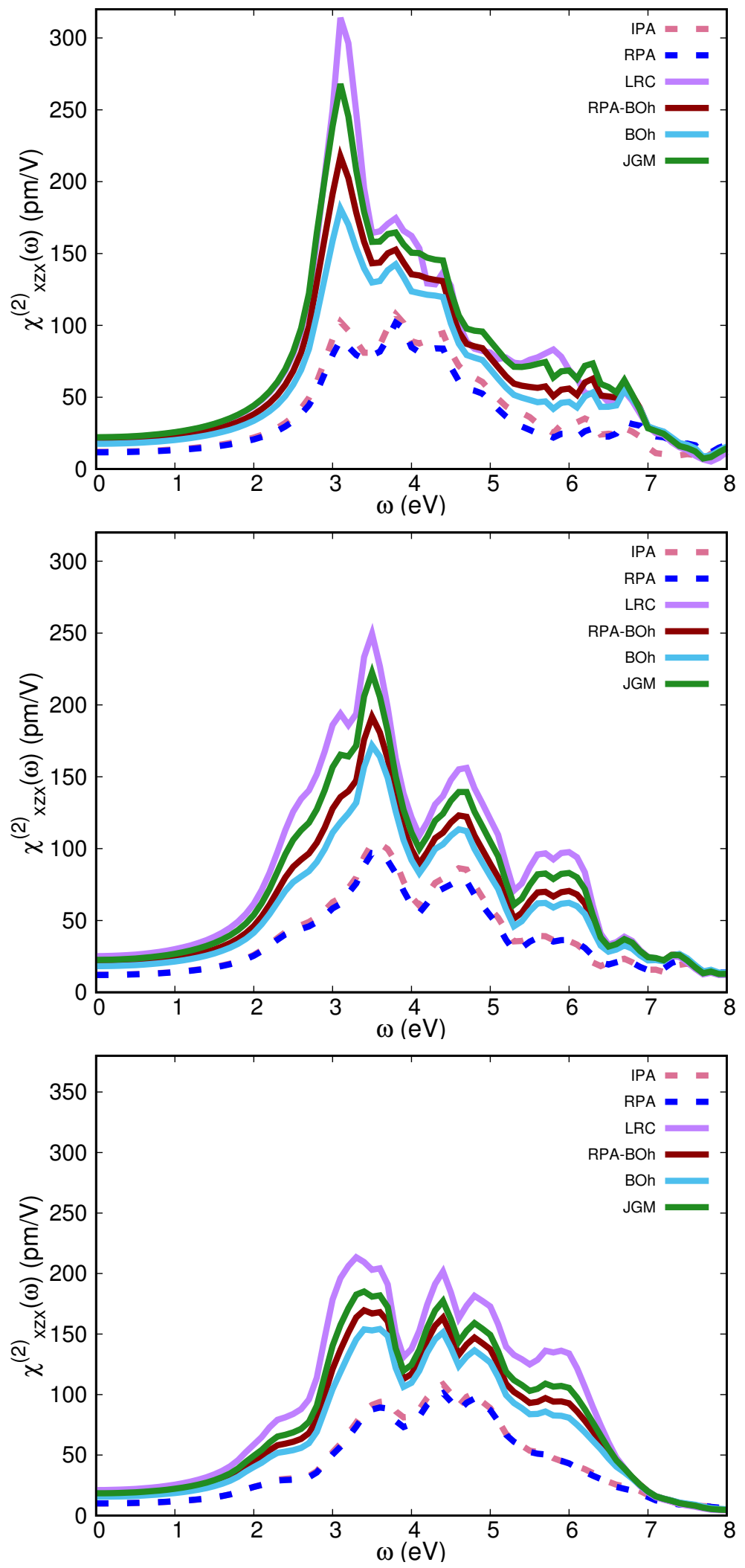

FIG. 9. SiC-2H (top), SiC-4H (center) and SiC-6H (bottom) : $\chi_{\mathrm{xzx}}^{(2)}(\omega)$ calculated with IPA, RPA, LRC, RPA-BOh, BOh, JGM kernels. 

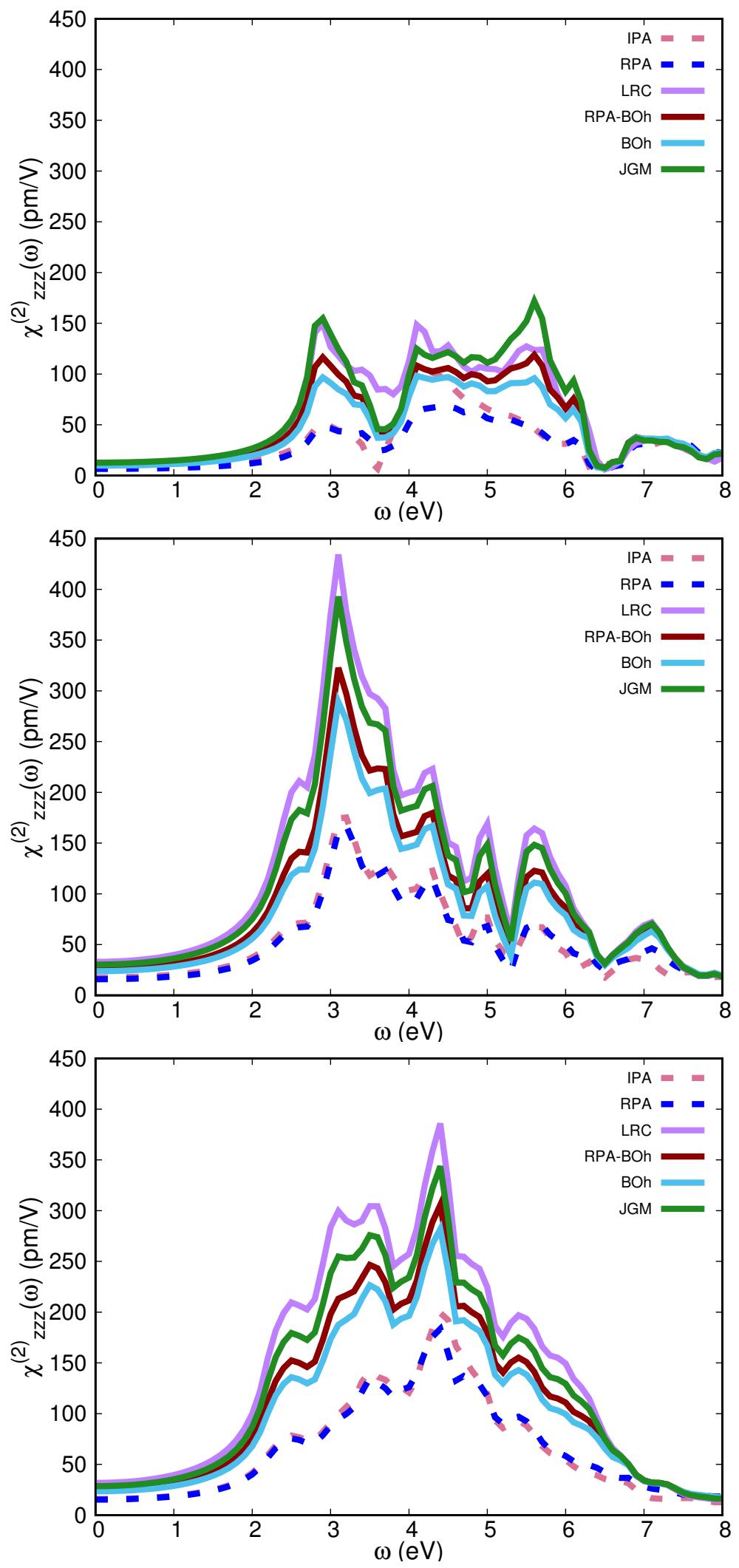

FIG. 10. SiC-2H (top), SiC-4H (center) and SiC-6H (bottom) : $\chi_{z z z}^{(2)}(\omega)$ calculated with IPA, RPA, LRC, RPA-BOh, BOh, JGM kernels. 
In the case of LRC, in order to obtain a better agreement with experiments, a smaller value of $\alpha^{\mathrm{LRC}}$ has to be taken : 0.2 instead of 0.5 . [38]

Next, we investigated the behaviour of the JGM kernel and we show $\chi_{\mathrm{xyz}}^{(2)}$ in Fig. (3). In Fig. (4), we compare IPA and RPA with the long-range kernels. In the case of the second-order response, the kernels do not shift noticeably the peaks but, instead cause a strong enhancement of the SHG intensity following the same trend observed in the linear response : $\alpha^{\mathrm{LRC}}>\alpha^{\mathrm{RPA}-\mathrm{BOh}}>\alpha^{\mathrm{BOh}}>\alpha^{\mathrm{JGM}}$.

\section{B. Cubic GaAs}

We used the experimental lattice constant $5.652 \AA$ and an energy cutoff of 50 Ha. We used 18522 shifted $k$ points in the Brillouin zone (BZ), 11 unoccupied states and $283 \mathbf{G}$ vectors for the wavefunctions. Crystal local-field effects are fully taken into account using $89 \mathbf{G}$ vectors. In order to simulate the quasiparticle energies we used a scissors shift of $0.8 \mathrm{eV}$ [60], and obtained a (minimum) direct electronic gap of $1.19 \mathrm{eV}$. In the case of JGM we used the experimental minimum electronic gap $E_{g}=1.52 \mathrm{eV}[61]$.

In Fig. (5), we show the effects of the long-range corrected kernels studied compared to RPA and IPA calculations. Both in IPA and RPA, the peaks at $3 \mathrm{eV}$ and $5 \mathrm{eV}$ are easily identified. However, the peak at $3 \mathrm{eV}$ is strongly underestimated compared to experiment because both IPA and RPA do not include excitonic effects. The long-range kernels can resolve this peak giving good agreement with experiments. Moreover, as already observed for $\mathrm{SiC}$, it is possible to quantify the effect of each kernel by considering that $\alpha^{\mathrm{LRC}}=0.22>\alpha^{\mathrm{JGM}}=0.20>\alpha^{\mathrm{RPA}-\mathrm{BOh}}=0.12>$ $\alpha^{\mathrm{BOh}}=0.10$. The same trend is found for $\epsilon_{1}$ (see Supplemental Information). The static value is shown in Tab. (II) and one clearly sees that no excitonic effect is needed to recover the experimental results. This holds for the linear and nonlinear coefficients.

The behaviour of the long-range kernels for the SHG is shown in Fig. (6). The general trend for all the kernels is to enhance the SHG intensity following the rule $\alpha^{\mathrm{LRC}}>\alpha^{\mathrm{JGM}}>\alpha^{\mathrm{RPA}-\mathrm{BOh}}>\alpha^{\mathrm{BOh}}$ which is the same trend we already described for $\epsilon_{1}$ and $\epsilon_{2}$.

Moreover, it is interesting to note that, as in the case of SiC, the effects of the SHG kernels do not depend on the frequency range. In the case of GaAs the kernels give noticeable effects only below $5 \mathrm{eV}$.

\section{Hexagonal SiC polytypes}

We studied the hexagonal SiC polytypes : $\mathrm{Si}-2 \mathrm{H}, \mathrm{Si}-4 \mathrm{H}$ and $\mathrm{Si}-6 \mathrm{H}$. For all the polytypes we used the experimental lattice constants, which for $\mathrm{Si}-2 \mathrm{H}$ is $\mathrm{a}=3.075 \AA$ and $\mathrm{c}=5.048 \AA$, for $\mathrm{Si}-4 \mathrm{H}$ is $\mathrm{a}=3.073 \AA$ and $\mathrm{c}=10.052 \AA$ and for Si- $6 \mathrm{H}$ is $\mathrm{a}=3.073 \AA$ and $\mathrm{c}=15.079 \AA$. We used an energy cutoff of $50 \mathrm{Ha}[59,62]$ and 5120 shifted $k$ points for $\mathrm{Si}-2 \mathrm{H}$ in the Brillouin zone (BZ), 3888 shifted $k$ points for Si- $4 \mathrm{H}$ and 4000 shifted $k$ points for SiC-6H. The number of unoccupied states are 54 for $\mathrm{SiC}-2 \mathrm{H}, 22$ for $\mathrm{SiC}-4 \mathrm{H}$ and 26 for $\mathrm{SiC}-6 \mathrm{H}$, and the number of $\mathbf{G}$ vectors for the wavefunctions is 497 for SiC-2H, 489 for SiC-4H and 493 G for SiC-6H. Crystal local-field effects are fully taken into account using 97 G vectors. 
In order to simulate the quasiparticle energies we used a scissors shift of $0.84 \mathrm{eV}$ [60]. For $\mathrm{SiC}-2 \mathrm{H}$ the minimum electronic gap is $3.01 \mathrm{eV}$ and the direct electronic gap is $4.72 \mathrm{eV}$. In the case of JGM we used the experimental minimum electronic gap $E_{g}=3.33 \mathrm{eV}$ [59]. For $\mathrm{SiC}-4 \mathrm{H}$ the minimum electronic gap is $3.03 \mathrm{eV}$ and the direct electronic gap is $4.16 \mathrm{eV}$. In the case of JGM, we used the experimental minimum electronic $E_{g}=3.26 \mathrm{eV}$ [59]. For SiC-6H the minimum electronic gap is $2.81 \mathrm{eV}$ and the direct electronic gap is $3.92 \mathrm{eV}$. In the case of JGM we used the experimental minimum electronic $E_{g}=3.02 \mathrm{eV}[59]$.

In Figs. $(7,8)$ we show the effects of the long-range corrected kernels RPA-BOh, BOh and JGM compared to RPA and IPA calculations for $\epsilon_{2, \mathrm{xx}}$ and $\epsilon_{2, \mathrm{zz}}$.

SiC-2H, SiC-4H and SiC-4H show the same trend, which can be quantified by observing that in the case of $\epsilon_{2, \mathrm{xx}}$ for $\mathrm{SiC}-2 \mathrm{H}$ we have $\alpha_{\mathrm{xx}}^{\mathrm{LRC}}=0.54>\alpha_{\mathrm{xx}}^{\mathrm{JGM}}=0.46>\alpha_{\mathrm{xx}}^{\mathrm{RPA}-\mathrm{BOh}}=0.40>\alpha_{\mathrm{xx}}^{\mathrm{BOh}}=0.32$, for SiC-4H we have $\alpha_{\mathrm{xx}}^{\mathrm{LRC}}=$ $0.51>\alpha_{\mathrm{xx}}^{\mathrm{JGM}}=0.43>\alpha_{\mathrm{xx}}^{\mathrm{RPA}-\mathrm{BOh}}=0.37>\alpha_{\mathrm{xx}}^{\mathrm{BOh}}=0.30$ and for SiC-6H we have $\alpha_{\mathrm{xx}}^{\mathrm{LRC}}=0.54>\alpha_{\mathrm{xx}}^{\mathrm{JGM}}=0.43>$ $\alpha_{\mathrm{xx}}^{\mathrm{RPA}-\mathrm{BOh}}=0.40>\alpha_{\mathrm{xx}}^{\mathrm{BOh}}=0.34$. The same is found for $\epsilon_{2, \mathrm{zz}}$ where we obtain for SiC-2H $\alpha_{\mathrm{zz}}^{\mathrm{LRC}}=0.50>\alpha_{\mathrm{zz}}^{\mathrm{JGM}}=$ $0.46>\alpha_{\mathrm{zZ}}^{\mathrm{RPA}-\mathrm{BOh}}=0.36>\alpha_{\mathrm{zz}}^{\mathrm{BOh}}=0.29$, for $\mathrm{SiC}-4 \mathrm{H} \alpha_{\mathrm{zz}}^{\mathrm{LRC}}=0.48>\alpha_{\mathrm{zZ}}^{\mathrm{JGM}}=0.43>\alpha_{\mathrm{zz}}^{\mathrm{RPA}-\mathrm{BOh}}=0.33>\alpha_{\mathrm{zz}}^{\mathrm{BOh}}=0.28$, and for for $\mathrm{SiC}-6 \mathrm{H} \alpha_{\mathrm{zZ}}^{\mathrm{LRC}}=0.50>\alpha_{\mathrm{zZ}}^{\mathrm{JGM}}=0.43>\alpha_{\mathrm{zz}}^{\mathrm{RPA}-\mathrm{BOh}}=0.36>\alpha_{\mathrm{zz}}^{\mathrm{BOh}}=0.31$.

Identical behaviour was found for $\epsilon_{1}$ (see Supplemental Information) and in its static value is shown in Tab. (III).

The performance of the long-range kernels for SHG is shown for $\chi_{\mathrm{xzx}}^{(2)}$ in Fig. (9) and for $\chi_{\mathrm{zzz}}^{(2)}$ in Fig. (10). In these cases the SHG is also enhanced but the main peaks are not noticeably shifted in energy. The enhancement exactly follows the trend of $\alpha$ found for the optical absorption.

The strong enhancement of SHG due to excitons was already observed in the two-dimensional hexagonal crystals $\mathrm{SiC}[19]$.

\section{Hexagonal $\mathrm{ZnO}$}

We used the experimental lattice constants $\mathrm{a}=3.258 \AA$ and $\mathrm{c}=5.220 \AA$ and an energy cutoff of 50 Ha. We used 5120 shifted $k$ points in the Brillouin zone (BZ), 32 unoccupied states and $487 \mathbf{G}$ vectors for the wavefunctions. Crystal local-field effects are fully taken into account using $99 \mathbf{G}$ vectors. In order to simulate the quasiparticle energies we used a scissors shift of $1.6 \mathrm{eV}$ [63]. The (minimum) direct electronic gap is $2.42 \mathrm{eV}$. In the case of JGM we used the experimental minimum electronic gap $E_{g}=3.44 \mathrm{eV}[64]$.

\begin{tabular}{|c|c|c|c|c|c|c|}
\hline & & & $\mathrm{ZnO}$ & & & \\
\hline & IPA & RPA & LRC & RPA-BOh & $\mathrm{BOh}$ & JGM \\
\hline$\epsilon_{1, \mathrm{xx}}$ & 4.28 & 4.16 & 5.08 & 5.16 & 4.92 & 4.84 \\
\hline$\alpha_{x}$ & & & 0.90 & 0.96 & 0.78 & 0.71 \\
\hline$\epsilon_{1, \mathrm{z}}$ & 4.27 & 4.16 & 5.09 & 5.16 & 4.93 & 4.84 \\
\hline$\alpha_{\mathrm{zz}}$ & & & 0.90 & 0.95 & 0.78 & 0.71 \\
\hline$\left|\chi_{\mathrm{xzX}}^{(2)}\right|$ & 0.735 & 0.34 & 0.73 & 0.77 & 0.65 & 0.61 \\
\hline$\left|\chi_{\mathrm{zzZ}}^{(2)}\right|$ & 31.21 & 30.01 & 64.85 & 68.43 & 57.71 & 54.07 \\
\hline
\end{tabular}

TABLE IV. Static values of $\epsilon_{1, \mathbf{x x}}, \epsilon_{1, \mathrm{zz}},\left|\chi_{\mathrm{xzx}}^{(2)}\right|[\mathrm{pm} / \mathrm{V}]$ and $\left|\chi_{\mathrm{zzz}}^{(2)}\right|[\mathrm{pm} / \mathrm{V}]$ for $\mathrm{ZnO}$. 

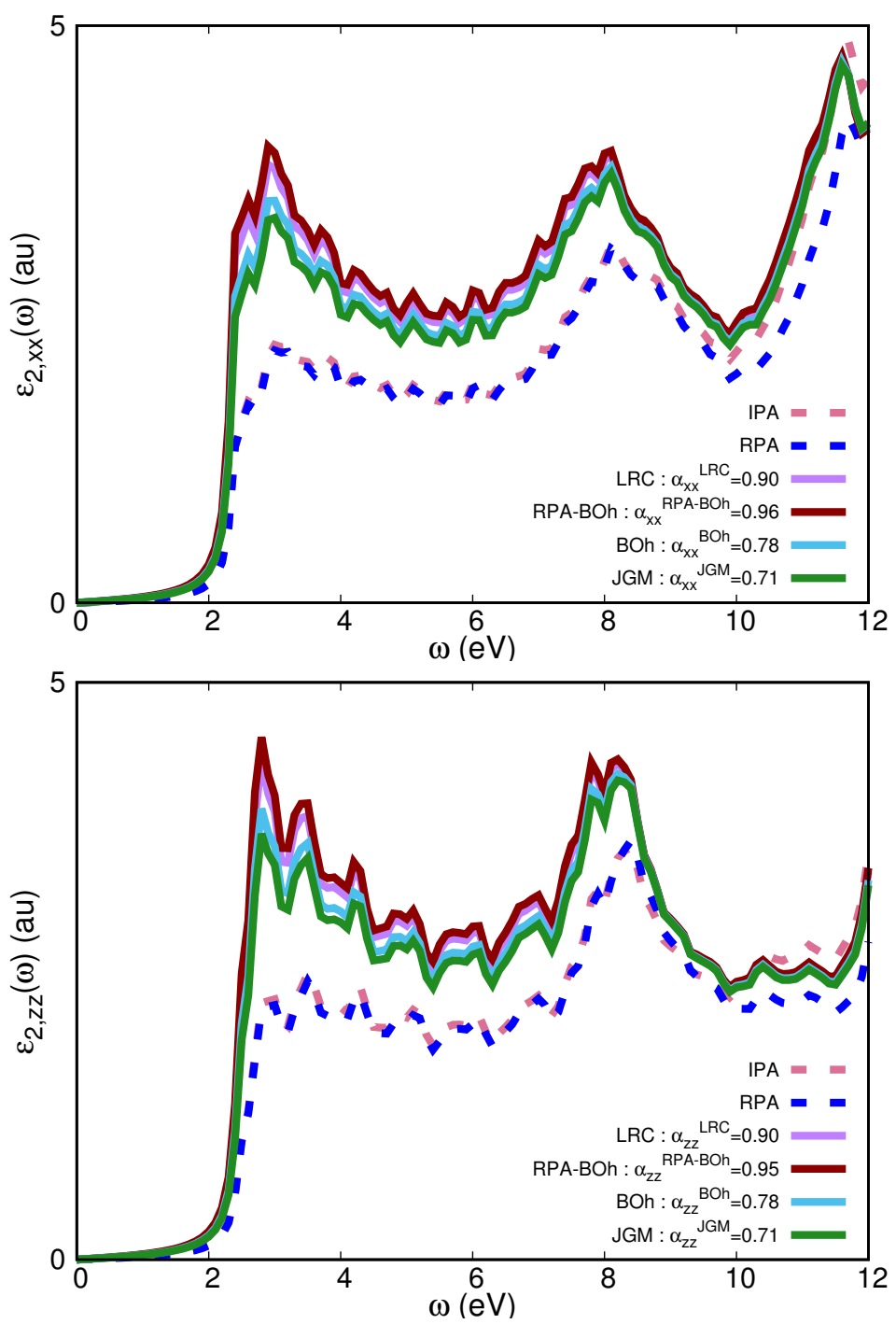

FIG. 11. ZnO : $\epsilon_{2, \mathrm{xx}}(\omega)$ and $\epsilon_{2, \mathrm{zz}}(\omega)$ calculated in IPA, RPA, LRC, RPA-BOh, BOh, JGM kernels.

In Fig. (11), we show the effects of the long-range corrected kernels LRC, RPA-BOh, BOh and JGM compared to RPA and IPA for $\epsilon_{2, \mathrm{xx}}$ and $\epsilon_{2, \mathrm{zz}}$. It is possible to quantify the effect of each kernels by considering that $\alpha_{\mathrm{xx}}^{\mathrm{RPA}-\mathrm{BOh}}=$ $0.96>\alpha_{\mathrm{xx}}^{\mathrm{LRC}}=0.90>\alpha_{\mathrm{xx}}^{\mathrm{BOh}}=0.78>\alpha_{\mathrm{xx}}^{\mathrm{JGM}}=0.71$. The same rule is followed by $\epsilon_{2, \mathrm{zz}}$ and $\alpha_{\mathrm{zz}}$ which values are reported in Tab. (IV) for each kernels. The trend of $\epsilon_{1}$ (see Supplemental Information) and in its static value is also shown in Tab. (IV).

It is interesting to note that in the case of $\mathrm{ZnO} \alpha^{\mathrm{LRC}}$ does not give the largest value and we suppose that this could be due to the break down of the model proposed by Botti et al. [38] to obtain $\alpha^{\text {LRC }}$. In fact, they already showed that this model can fail for materials with a small dielectric constant. In this case they suggested that the value of $\alpha^{\mathrm{LRC}}$ should be higher, which, however, would then deteriorate the low-energy part of the spectrum.

The behaviour of the long-range kernels for SHG is shown for $\chi_{\mathrm{xzx}}^{(2)}$ and $\chi_{\mathrm{zzz}}^{(2)}$ in Fig. (12) and confirms that excitons enhance the SHG without affecting the energy position of the peaks.

Recently, the optical properties of $\mathrm{ZnO}$ have also been calculated in the BSE framework, showing a good agreement 

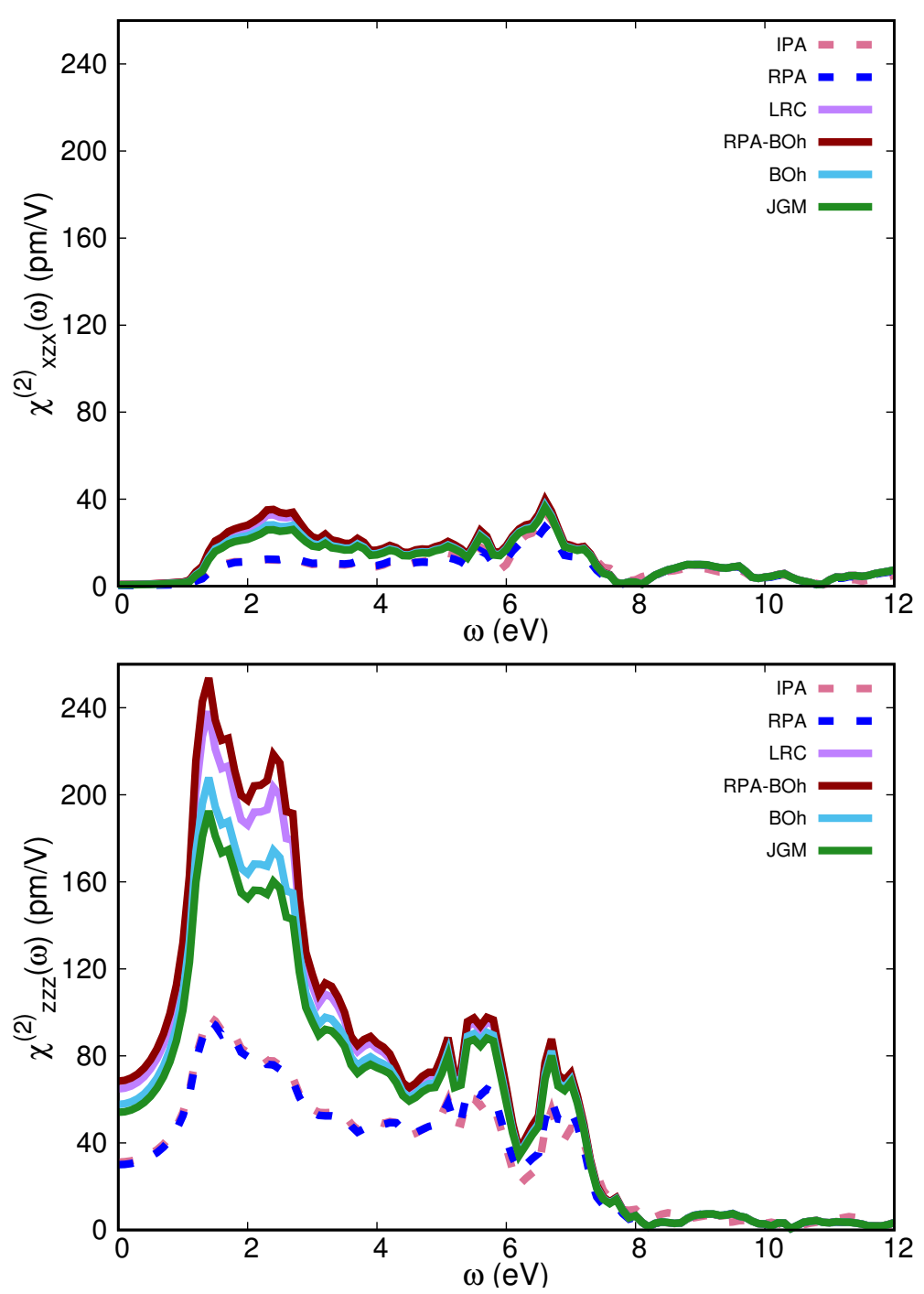

FIG. 12. $\mathrm{ZnO}: \chi_{\mathrm{xzx}}^{(2)}$ and $\chi_{\mathrm{zzz}}^{(2)}$ calculated with IPA, RPA, LRC, RPA-BOh, BOh and JGM kernels.

\begin{tabular}{ccccccc}
\hline \hline \multicolumn{7}{c}{ ZnGeP } \\
& IPA & RPA & LRC & RPA-BOh & BOh & JGM \\
\hline$\epsilon_{2, \mathrm{xx}}$ & 10.27 & 9.61 & 10.99 & 10.61 & 10.45 & 13.27 \\
$\alpha_{\mathrm{xx}}$ & & & 0.29 & 0.15 & 0.13 & 0.43 \\
$\left|\chi_{\mathrm{xyz}}^{(2)}\right|$ & 137.15 & 135.15 & 250.16 & 189.21 & 179.24 & 380.29 \\
\hline \hline
\end{tabular}

TABLE V. Static values of $\epsilon_{1, \mathrm{xx}}$ and $\left|\chi_{\mathrm{xyz}}^{(2)}\right|[\mathrm{pm} / \mathrm{V}]$ for ZnGeP. The $\alpha$-parameter is reported for the long-range corrected kernels.

with TDDFT with long-range kernels. [65]

\section{E. Tetragonal $\mathbf{Z n}_{2} \mathbf{G e}_{2} \mathbf{P}_{4}$}

We used the experimental lattice constants $\mathrm{a}=5.46 \AA$ and $\mathrm{c}=10.83 \AA$ and an energy cutoff of $50 \mathrm{Ha}$ [66, 67]. We used 3456 shifted $k$ points in the Brillouin zone (BZ), 64 unoccupied states and 485 G vectors for the wavefunctions. Crystal local-field effects are fully taken into account using $97 \mathbf{G}$ vectors. In order to simulate the quasiparticle 


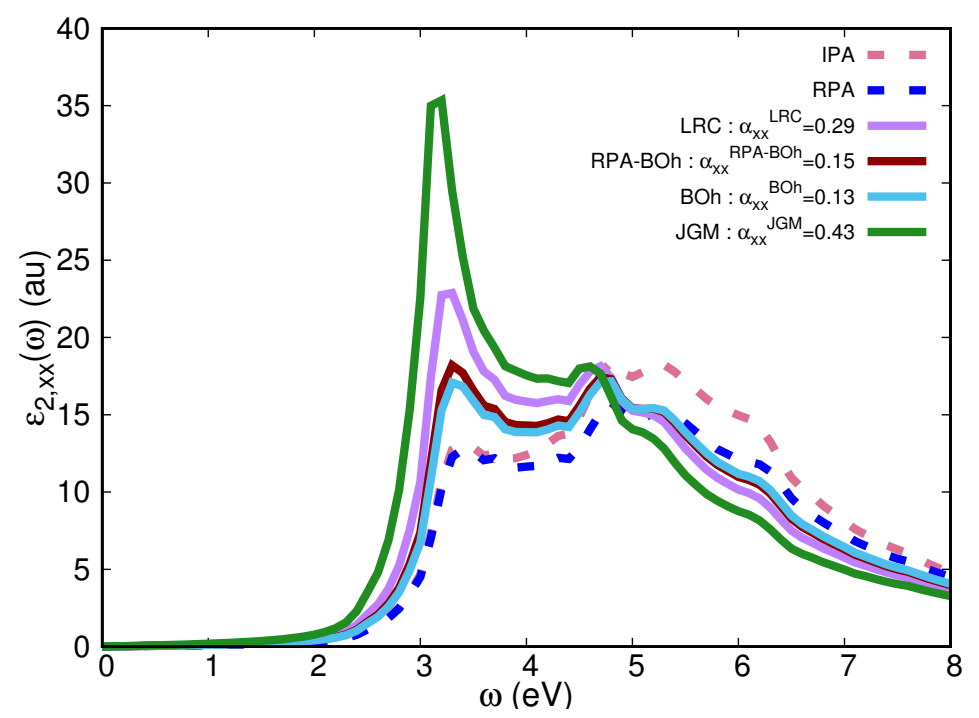

FIG. 13. ZnGeP : $\epsilon_{2, \mathrm{xx}}(\omega)$ calculated in IPA, RPA, LRC, RPA-BOh, BOh, JGM kernels.

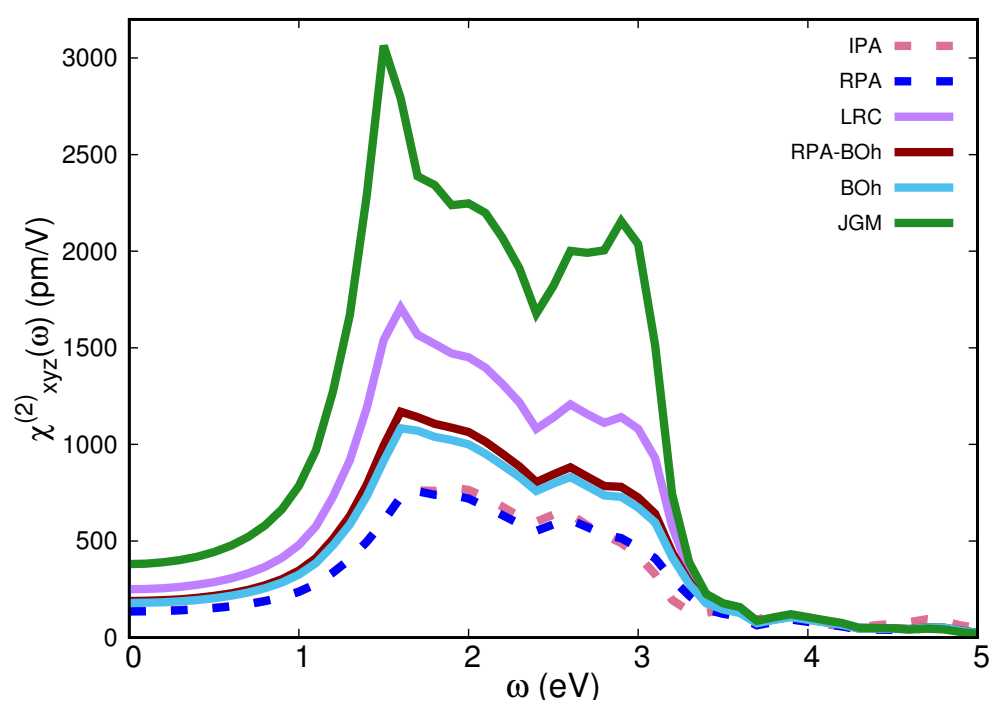

FIG. 14. ZnGeP : $\chi_{\mathrm{xyz}}^{(2)}$ calculated with IPA, RPA, LRC, RPA-BOh, BOh and JGM kernels.

energies we used a scissors shift of $1.04 \mathrm{eV}$ [60]. The minimum electronic gap is $2.23 \mathrm{eV}$ and the direct electronic gap is $2.34 \mathrm{eV}$. In the case of JGM we used the experimental minimum electronic gap $E_{g}=2.34 \mathrm{eV}$ [68].

In Fig. (13), we show the effects of the long-range corrected kernels LRC, RPA-BOh, BOh and JGM compared to RPA and IPA calculations for $\epsilon_{2, \mathrm{xx}}$. We found $\alpha_{\mathrm{xx}}^{\mathrm{JGM}}=0.43>\alpha_{\mathrm{xx}}^{\mathrm{LRC}}=0.29>\alpha_{\mathrm{xx}}^{\mathrm{RPA}-\mathrm{BOh}}=0.15>\alpha_{\mathrm{xx}}^{\mathrm{BOh}}=0.13$. The same trend is found for $\epsilon_{1}$ (see Supplemental Information) and in its static value is shown in Tab. (V).

The behaviour of the long-range kernels for SHG is shown for $\chi_{x y z}^{(2)}$ in Fig. (14). Here again, excitonic effects contribute to enhance the SHG signal. However, the enhancement is particularly strong in the case of the JGM concerning a low energy peak around $3 \mathrm{eV}$ which is set too low in IPA and RPA. The other long-range kernels LRC, RPA-BOh and BOh are able to increase this peak with respect IPA and RPA, but its intensity is still very low in comparison with JGM. A similar behaviour of the JGM versus LRC kernel was already observed by Grüning and 
Attaccalite [69] for bulk zinc chalcogenides ZnS, ZnSe and ZnTe.

\section{CONCLUSIONS}

The role of excitons in second-harmonic generation has been investigated using different long-range exchangecorrelation kernels (empirical LRC, Bootstrap RPA-BOh and BOh, and JGM) in the framework of TDDFT. Through the calculation of $\chi^{(2)}$ and $\epsilon_{\mathrm{M}}$ we determined absorption and SHG spectra for different electric-field polarizations and crystal symmetries : cubic zincblende, hexagonal wurtzite and tetragonal symmetry. Our study indicates that excitons play an important role in $\chi^{(2)}$. They induce a strong enhancement of the SHG signal but do not noticeably shift the peaks in the spectra even though a large change in position of the spectral peaks is observed for $\epsilon_{\mathrm{M}}$. Moreover, the enhancement follows a simple trend which depends on the magnitude of the long-range corrected $\alpha$-parameter. We observe that as a general rule $\alpha^{\mathrm{RPA}-\mathrm{BOh}}>\alpha^{\mathrm{RPA}}$, but a material dependence on $\alpha^{\mathrm{JGM}}$ and $\alpha^{\mathrm{LRC}}$ is obtained, which does not permit to find an absolute trend for the $\alpha$-parameter for the materials studied. Moreover, comparing with the available experimental results, we found that it is not possible to identify which is the "best" kernel. The lack of frequency dependence in the $\alpha$-parameter implies that the optical properties can be better described in some energy regions than in others. At the moment, most of the calculations of SHG are performed in IPA which can give very approximate results. As we show that excitonic effects can be very strong for these systems, we believe that going beyond IPA is therefore necessary to accurately interpret experiments.

\section{ACKNOWLEDGEMENTS}

This work was performed using HPC resources from GENCI-IDRIS Grant No. 090544.

[1] Y. R. Shen, Principles Nonlinear Optics, (Wiley-Interscience, New York, 1984).

[2] E. Luppi and V. Véniard, Semiconductor Science and Technology 31, 123002 (2016).

[3] M. Cazzanelli, F. Bianco, E. Borga, G. Pucker, M. Ghulinyan, E. Degoli, E. Luppi, V. Véniard, S. Ossicini, D. Modotto, R. P. S. Wabnitz, and L. Pavesi, Nature Materials 11, 148 (2012).

[4] M. Grüning and C. Attaccalite, Phys. Rev. B 89, 081102 (2014).

[5] A.-M. Janner, H. T. Jonkman, and G. A. Sawatzky, Phys. Rev. B 63, 085111 (2001).

[6] M. Lafrentz, D. Brunne, A. V. Rodina, V. V. Pavlov, R. V. Pisarev, D. R. Yakovlev, A. Bakin, and M. Bayer, Phys. Rev. B 88, 235207 (2013).

[7] K. L. Seyler, J. R. Schaibley, P. Gong, P. Rivera, A. M. Jones, S. Wu, J. Yan, D. G. Mandrus, W. Yao, and X. Xu, Nature Nanotech. 10, 407 (2015).

[8] C. Janisch, Y. Wang, D. Ma, N. Mehta, A. L. Elías, N. Perea-López, M. Terrones, V. Crespi, and Z. Liu, Sci. Rep. 4, 5530 (2014).

[9] L. M. Malard, T. V. Alencar, A. P. M. Barboza, K. F. Mak, and A. M. de Paula, Phys. Rev. B 87, 201401 (2013). 
[10] Y. Li, Y. Rao, K. F. Mak, Y. You, S. Wang, C. R. Dean, and T. F. Heinz, Nano Lett. 13, 3329 (2013).

[11] T. G. Pedersen, Phys. Rev. B 92, 235432 (2015).

[12] E. Luppi, E. Degoli, M. Bertocchi, S. Ossicini, and V. Véniard, Phys. Rev. B 92, 075204 (2015).

[13] M. Lafrentz, D. Brunne, B. Kaminski, V. V. Pavlov, A. V. Rodina, R. V. Pisarev, D. R. Yakovlev, A. Bakin, and M. Bayer, Phys. Rev. Lett. 110, 116402 (2013).

[14] V. V. Pavlov, A. M. Kalashnikova, R. V. Pisarev, I. Sänger, D. R. Yakovlev, and M. Bayer, Phys. Rev. Lett. 94, 157404 (2005).

[15] D. Brunne, M. Lafrentz, V. V. Pavlov, R. V. Pisarev, A. V. Rodina, D. R. Yakovlev, and M. Bayer, Phys. Rev. B 92, $085202(2015)$.

[16] A.-M. Janner, R. Eder, B. Koopmans, H. T. Jonkman, and G. A. Sawatzky, Phys. Rev. B 52, 17158 (1995).

[17] V. G. Sala, S. Dal Conte, T. A. Miller, D. Viola, E. Luppi, V. Véniard, G. Cerullo, and S. Wall, Phys. Rev. B 94, 014430 (2016).

[18] S. Bergfeld and W. Daum, Phys. Rev. Lett. 90, 036801 (2003).

[19] C. Attaccalite, A. Nguer, E. Cannuccia, and M. Gruning, Phys. Chem. Chem. Phys. 17, 9533 (2015).

[20] H. Zeng, G.-B. Liu, J. Dai, Y. Yan, B. Zhu, R. He, L. Xie, S. Xu, X. Chen, W. Yao, and X. Cui, 3, 1608 (2013).

[21] N. Bloembergen, Nonlinear Optics, (Benjamin Press, New York, 1965).

[22] E. Luppi, H. Hübener, and V. Véniard, Phys. Rev. B 82, 235201 (2010).

[23] R. Del Sole and E. Fiorino, Phys. Rev. B 29, 4631 (1984).

[24] G. Onida, L. Reining, and A. Rubio, Rev. Mod. Phys. 74, 601 (2002).

[25] E. C. Chang, E. L. Shirley, and Z. H. Levine, Phys. Rev. B 65, 035205 (2001).

[26] R. Leitsmann, W. G. Schmidt, P. H. Hahn, and F. Bechstedt, Phys. Rev. B 71, 195209 (2005).

[27] L. Sponza, H. Amara, F. m. c. Ducastelle, A. Loiseau, and C. Attaccalite, Phys. Rev. B 97, 075121 (2018).

[28] P. Cudazzo, L. Sponza, C. Giorgetti, L. Reining, F. Sottile, and M. Gatti, Phys. Rev. Lett. 116, 066803 (2016).

[29] E. Rebolini and J. Toulouse, The Journal of Chemical Physics 144, 094107 (2016), https://doi.org/10.1063/1.4943003.

[30] A. Riefer and W. G. Schmidt, Phys. Rev. B 96, 235206 (2017).

[31] C. Attaccalite and M. Grüning, Phys. Rev. B 88, 235113 (2013).

[32] I. Souza, J. Íñiguez, and D. Vanderbilt, Phys. Rev. B 69, 085106 (2004).

[33] E. Luppi, H. Hübener and V. Véniard, J. Chem. Phys., accepted.

[34] E. Runge and E. K. U. Gross, Phys. Rev. Lett. 52, 997 (1984).

[35] E. K. U. Gross and W. Kohn, Phys. Rev. Lett. 55, 2850 (1985).

[36] E. Gross, J. Dobson, and M. Petersilka, in Density-functional theory time-dependent phenomena, Topics in Current Chem., Vol. 181 (Springer, 1996) pp. 81-172.

[37] W. G. Aulbur, L. Jönsson, and J. W. Wilkins, Phys. Rev. B 54, 8540 (1996).

[38] S. Botti, F. Sottile, N. Vast, V. Olevano, L. Reining, H.-C. Weissker, A. Rubio, G. Onida, R. D. Sole, and R. W. Godby, Phys. Rev. B 69, 155112 (2004).

[39] L. Reining, V. Olevano, A. Rubio, and G. Onida, Phys. Rev. Lett. 88, 066404 (2002).

[40] S. Sharma, J. K. Dewhurst, A. Sanna, and E. K. U. Gross, Phys. Rev. Lett. 107, 186401 (2011).

[41] S. Rigamonti, S. Botti, V. Veniard, C. Draxl, L. Reining, and F. Sottile, Phys. Rev. Lett. 117, 159702 (2016).

[42] P. E. Trevisanutto, A. Terentjevs, L. A. Constantin, V. Olevano, and F. D. Sala, Phys. Rev. B 87, 205143 (2013).

[43] Y.-M. Byun and C. A. Ullrich, Phys. Rev. B 95, 205136 (2017). 
[44] H. Hübener, E. Luppi, and V. Véniard, Phys. Rev. B 83, 115205 (2011).

[45] L. A. Constantin and J. M. Pitarke, Phys. Rev. B 75, 245127 (2007).

[46] J. A. Berger, Phys. Rev. Lett. 115, 137402 (2015).

[47] E. Luppi, H.-C. Weissker, S. Bottaro, F. Sottile, V. Veniard, L. Reining, and G. Onida, Phys. Rev. B 78, 245124 (2008).

[48] N. Troullier and J. L. Martins, Phys. Rev. B 43, 1993 (1991).

[49] (), x. Gonze and B. Amadon and P.-M. Anglade and J.-M. Beuken and F. Bottin and P. Boulanger and F. Bruneval and D. Caliste and R. Caracas and M. Cote and T. Deutsch and L. Genovese and Ph. Ghosez and M. Giantomassi and S. Goedecker and D.R. Hamann and P. Hermet and F. Jollet and G. Jomard and S. Leroux and M. Mancini and S. Mazevet and M. J. T. Oliveira and G. Onida and Y. Pouillon and T. Rangel and G.-M. Rignanese and D. Sangalli and R. Shaltaf and M. Torrent and M. J. Verstraete and G. Zerah and J.W. Zwanziger, Computer Phys. Commun. 180, 2582-2615 (2009).

[50] (), x. Gonze and G.-M. Rignanese and M. Verstraete and J.-M. Beuken and Y. Pouillon and R. Caracas and F. Jollet and M. Torrent and G. Zerah and M. Mikami and Ph. Ghosez and M. Veithen and J.-Y. Raty and V. Olevano and F. Bruneval and L. Reining and R. Godby and G. Onida and D. R. Hamann and D.C. Allan, Zeit. Kristallogr. 220, 558-562 (2005).

[51] (), aBINIT code is a common project the Université Catholique de Louvain, Corning Incorporated, and other contributors (URL http://www.abinit.org).

[52] N. Tancogne-Dejean, B. S. Mendoza, and V. Véniard, Phys. Rev. B 90, 035212 (2014).

[53] E. Luppi, H. Hübener, and V. Véniard, Phys. Rev. B 82, 235201 (2010).

[54] E. Luppi and H. Hübner and V. Veniard, to be submitted to Comput. Phys. Commun.

[55] S. Logothetidis and J. Petalas, Journal of Applied Physics 80, 1768 (1996), https://doi.org/10.1063/1.362975.

[56] D. Aspnes and A. A. Studna, Phys. Rev. B 27, 985 (1983).

[57] L. Pavesi and M. Guzzi, Journal of Applied Physics 75, 4779 (1994), https://doi.org/10.1063/1.355769.

[58] N. Suzuki and K. Tada, Japanese Journal of Applied Physics 23, 1011 (1984).

[59] B. Baumeier, P. Krüger, and J. Pollmann, Phys. Rev. B 73, 195205 (2006).

[60] V. I. Gavrilenko and F. Bechstedt, Phys. Rev. B 54, 13416 (1996).

[61] F. Tran and P. Blaha, Phys. Rev. Lett. 102, 226401 (2009).

[62] P. E. Van Camp, V. E. Van Doren, and J. T. Devreese, Phys. Rev. B 44, 9056 (1991).

[63] A. Berger, HDR thesis, Laboratoire de Chimie et Physique Quantique, 2017.

[64] A. Riefer, N. Weber, J. Mund, D. R. Yakovlev, M. Bayer, A. Schindlmayr, C. Meier, and W. G. Schmidt, Journal of Physics: Condensed Matter 29, 215702 (2017).

[65] X. Zhang and A. Schleife, Phys. Rev. B 97, 125201 (2018).

[66] S. Limpijumnong, W. R. L. Lambrecht, and B. Segall, Phys. Rev. B 60, 8087 (1999).

[67] P. Paufler, Crystal Research and Technology 23, 1360 (1988), https://onlinelibrary.wiley.com/doi/pdf/10.1002/crat.2170231029.

[68] J. E. Jaffe and A. Zunger, Phys. Rev. B 30, 741 (1984).

[69] M. Grüning and C. Attaccalite, Phys. Chem. Chem. Phys 18, 2117 (2016). 